\title{
Broad-temperature range spectroscopy of the two-centre modular redox metalloprotein Desulfovibrio desulfuricans desulfoferrodoxin
}

\author{
Niels H. Andersen, ${ }^{a}$ Sven E. Harnung, ${ }^{b}$ Ib Trabjerg, ${ }^{b}$ Isabel Moura, ${ }^{c}$ José J. G. Moura ${ }^{c}$ \\ and Jens Ulstrup ${ }^{a}$ \\ a Department of Chemistry, Building 207, Technical University of Denmark, \\ DK-2800 Lyngby, Denmark \\ ${ }^{b}$ Department of Chemistry, University of Copenhagen, Universitetsparken 5, \\ DK-2100 Copenhagen, Denmark \\ ${ }^{c}$ Departamento de Química and, Centro de Química Fina e Biotecnologia, \\ Faculdade de Ciéncas e Tecnologia, Universidade Nova de Lisboa, 2825-11 Caparica, Portugal
}

Received 1st April 2003, Accepted 2nd July 2003

First published as an Advance Article on the web 24th July 2003

The electronic-vibrational couplings of the two-centre non-heme iron protein Desulfovibrio desulfuricans desulfoferrodoxin (DFx) in three oxidation states, i.e. fully oxidised (grey), half-oxidised (pink), and fully reduced (colourless), have been investigated by variable temperature (VT) UV/VIS, MCD, CD, and EPR spectroscopy. The UV/VIS spectra of grey DFx at room temperature is characterised by broad charge transfer (CT) transitions associated with oxidised centre I (495 and $368 \mathrm{~nm}$ ) and II (335 and $635 \mathrm{~nm}$ ). The transitions are resolved at $78 \mathrm{~K}$, substantiated by VT-MCD and -CD. The data offer novel information about the electronic-vibrational couplings of the transitions. Multiphonon bandshape analysis discloses strong contributions from both local Fe-S and S-C stretching and solvent/protein modes. A number of transitions are blue- or red-shifted compared with monomeric desulforedoxin, superoxide reductase or dismutase, and cloned Desulfovibrio vulgaris DFx fragments. Conversion from grey to pink DFx is accompanied by drastic electronic-vibrational changes of both centres. The data suggest that electron transfer and optical CT-transitions of DFx are controlled by environmental reorganization in the whole region between the metal centres.

\section{Introduction}

Electron transfer (ET) has long been in strong focus as one of the most important elementary reaction steps in chemistry and biology. Both microscopic features and the organization of biological ET chains have been mapped in detail, and broad understanding of the central controlling factors achieved. ${ }^{1,2}$ A crucial notion is long-range ET between localized metal centres, for which the spatial separation significantly exceeds the structural extension of the centres and their local environment. A second feature is that ET function is frequently undertaken by proteins containing several redox centres, which mutually "communicate". This notion implies not only that the centres exchange electrons but also that they affect each other, by electrostatic interactions, or via the conformational protein system. The comprehensive mapping of cooperativity in the four-heme cytochromes $c_{3}$ has, for example led to detailed microscopic understanding of this protein. ${ }^{3,4}$ The large number of inter-heme contacts, and microscopic redox potentials is, however, prohibitive for complete microscopic mapping. In this respect redox metalloproteins with two centres offer special perspectives. These proteins are prototypes for multi-centre electron transport, but structurally and functionally simple enough that complete thermodynamic, spectral, and kinetic mapping is in principle feasible. This offers microscopic insight into both cooperative multi-centre ET and spectral interactions between the communicating centres, as reflected in $\mathrm{CT}$ and other spectral transitions. ${ }^{5}$

In this report we address the modular two-centre non-heme iron redox protein DFx from the sulfate reducing bacterium Desulfovibrio desulfuricans $(D d) .{ }^{6}$ Our objective is to disclose details of the electronic structure and electronic-vibrational couplings of the two centres, as well as changes of these couplings in the different oxidation states. DFx is engaged in monomer-dimer equilibria. ${ }^{7}$ These are apparently $\mathrm{Ca}^{2+}$. independent ${ }^{8}$ but $\mathrm{Ca}^{2+}$-ions are indispensable when crystallising $\mathrm{DFx},{ }^{9}$ and both monomer-dimer equilibria and conformational details may still be influenced by the presence of $\mathrm{Ca}^{2+}$-ions. The monomer is a $13.9 \mathrm{kDa}, 125$-residue protein. ${ }^{10}$ Well separated oxidation potentials of the two metal centres $(+4 \pm 10 \mathrm{mV}$ and $+240 \pm 10 \mathrm{mV}$ vs. SHE $),{ }^{11}$ ensure that three different oxidation states, grey, pink, and colourless, i.e. $\mathrm{Fe}_{\mathrm{I}}(\mathrm{III}) \mathrm{Fe}_{\text {II }}$ (III), $\mathrm{Fe}_{\mathrm{I}}(\mathrm{III}) \mathrm{Fe}_{\text {III }}$ (II) and $\mathrm{Fe}_{\mathrm{I}}(\mathrm{II}) \mathrm{Fe}_{\text {II }}$ (II), can be characterised. In the following " $\mathrm{Fe}_{\mathrm{I}}$ " and " $\mathrm{Fe}_{\text {II }}$ " refer to centre I (low-potential) and II (high-potential), respectively. Comprehensive room temperature (RT) spectroscopic (UV/VIS, Mössbauer, EPR, and resonance Raman spectroscopy) ${ }^{6,11}$ and a high-resolution three-dimensional $\mathrm{X}$-ray crystal structure ${ }^{12}$ have disclosed many structural details (Figs. 1 and 2). $\mathrm{Fe}_{\mathrm{I}}$, located in a 34-residue domain, resembles the rubredoxin-like Desulfovibrio gigas $(\mathrm{Dg})$ desulforedoxin (Dx) $\mathrm{Fe}(\mathrm{S}-\mathrm{Cys})_{4}$-centre (Cys9, Cys12, Cys28 and Cys29), with two adjacent Cys-ligands

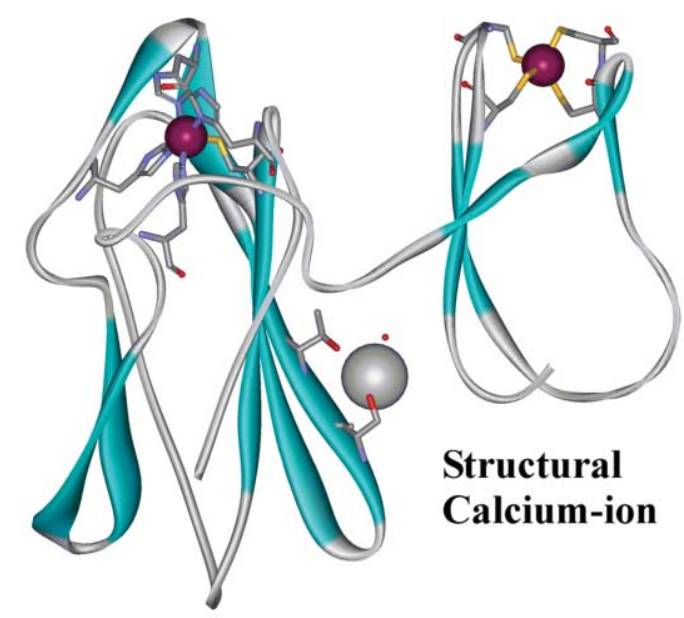

Fig. 1 Three-dimensional ribbon structure of the two domains of Desulfovibrio desulfuricans DFx. Right and left: domain I and II respectively. Data source: 1DFX.pdb at http://www.biochem.ucl. ac.uk/ bsm/pdbsum. Graphic representation: Weblab Viewer Pro. 


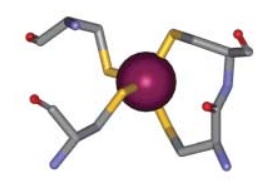

Centre I

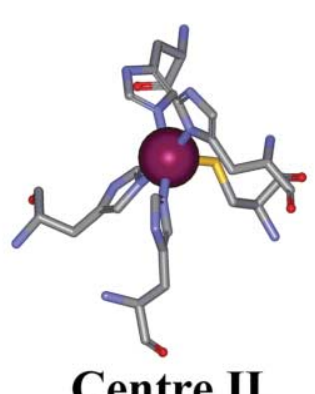

Centre II

Fig. 2 Three-dimensional structure of the two iron centres in Desulfovibrio desulfuricans DFx. Left: centre I. Right: centre II. Data source: 1dfx.pdb at http://www.biochem.ucl.ac.uk/bsm/pdbsum. Graphic representation: Weblab Viewer Pro.

in the coordination sphere. ${ }^{13} \mathrm{Fe}_{\mathrm{II}}$ is located in a 88 -residue domain $21.6 \AA$ from $\mathrm{Fe}_{\mathrm{I}}$. In this Fe-centre, $\mathrm{Fe}\left(\mathrm{N}_{\varepsilon}-\mathrm{His}\right)_{3^{-}}$ $\left(\mathrm{N}_{\delta}-\mathrm{His}\right)$ (S-Cys), iron is coordinated to four histidines and an apical Cys in a distorted square pyramidal structure (His48, His68, His74, His118 and Cys115). This novel type of nonheme iron centre has been encountered in other proteins and reported to display superoxide dismutase (SOD) and reductase (SOR) activity. ${ }^{14-16}$ The N- and C-terminal fragments (modules) of DFx from Desulfovibrio vulgaris $(D v)$ representing domain I and II, respectively, have also been expressed in Escherichia coli and characterised spectroscopically. ${ }^{17}$ High-resolution X-ray structures are available for Pyrococcus furiosus $(P f) \mathrm{SOR},{ }^{18}$ to which structural and spectral reference will be important in the following. ${ }^{19}$ The DFx structure shows a homo-dimer form where two DFx molecules are related by a two-fold symmetry axis. The inter-domain region between the two DFx molecules holds six structural water molecules in an ordered hydrogen bond network in which a $\mathrm{Ca}^{2+}$-ion is involved, indicative of enzyme and trigger function. The $\mathrm{Ca}^{2+}$-ion which lies on the twofold axis of the DFx-dimer is ligated twice by Ser87 (both O and $\left.\mathrm{O}_{\gamma}\right)$, Thr89 $\left(\mathrm{O}_{\gamma}\right)$ and a water molecule. ${ }^{12}$ DFx shows, finally, an interesting surface electrostatic charge distribution. The overall surface charge density is high and negative, but two patches of high positive charge are localized ${ }^{20}$ close to $\mathrm{Fe}_{\text {II }}$ and may serve a molecular recognition function.

Distinct ligand-to-metal CT bands dominate the UV/VIS spectra of the two centres. The protein is therefore well suited for addressing electronic-vibrational coupling on optical excitation, and perhaps interactions between the centres. The present study first addresses the CT spectral properties of DFx in the three states by UV/VIS spectroscopy in a broad temperature range. These data provide a spectral resolution, which has warranted analysis based on multiphonon optical bandshape theory ${ }^{2,21}$ for each of the 45 spectra covering the temperature range from 78-298 K. VT-MCD and CD spectra of DFx in the three oxidation states have, secondly, been recorded. The electronic-vibrational coupling is strong, and suggests that conformational sub-states are present. Major structural/ conformational changes in both centres also accompany the conversion from pink to either grey or colourless DFx.

\section{Experimental}

Desulfovibrio desulfuricans was grown and DFx isolated and purified as described. ${ }^{6}$ Stock solutions $\left(\gamma_{\mathrm{DFx}}=13.5 \mathrm{mg} \mathrm{ml}^{-1}\right.$, $\left.A_{280} / A_{495}=5.95\right)$ in TRIS/HCl buffer (2-amino-1,3-dihydroxy2-hydroxymethylpropane) $\mathrm{pH} 7.6$ were prepared and stored at $-80^{\circ} \mathrm{C}$.

Chemicals for buffers and protein handling, i.e. $\mathrm{Na}_{2} \mathrm{HPO}_{4}$. $2 \mathrm{H}_{2} \mathrm{O}, \mathrm{NaH}_{2} \mathrm{PO}_{4} \cdot 2 \mathrm{H}_{2} \mathrm{O},\left(\mathrm{CH}_{3}\right)_{2} \mathrm{AsO}_{2} \mathrm{H}$ (cacodylicacid), $\mathrm{Na}_{2} \mathrm{~S}_{2} \mathrm{O}_{4}$. $2 \mathrm{H}_{2} \mathrm{O}$ (sodium dithionite), $\mathrm{NaOH}$, and $\mathrm{CaO}$ were analytical grade. Water was Milli-Q (Milli-Q Housing). The amounts of components needed for buffer preparations were calculated using the Davies equation for activity coefficient correction. All

buffers were prepared as below with an ionic strength $I=30$ $\mathrm{mm}$, and $\mathrm{pH}=7.0$ before mixing with glycerol. The latter was added as a glassing agent in the ratio $2: 1(\mathrm{v} / \mathrm{v})$. Solutions were vacuum-filtered through a $45 \mu \mathrm{m}$ millipore filter and stored at $4{ }^{\circ} \mathrm{C}$.

Sodium cacodylate ( $\mathrm{NaCcd}$ ): dissolution of $2.394 \mathrm{~g}$ cacodylic acid in water, followed by $0.600 \mathrm{~g} \mathrm{NaOH}$ in a $500 \mathrm{ml}$ volumetric flask.

Calcium cacodylate $(\mathrm{CaCcd})$ : sonication of $\mathrm{CaO}$-powder $(0.2804 \mathrm{~g})$ in cacodylic acid (1.594 g) solution. After dissolution the volume was adjusted to $500 \mathrm{ml}$.

Phosphate buffer: dissolution of $0.1349 \mathrm{~g} \mathrm{Na}_{2} \mathrm{HPO}_{4} \cdot 2 \mathrm{H}_{2} \mathrm{O}$ and $0.1133 \mathrm{~g} \mathrm{NaH}{ }_{2} \mathrm{PO}_{4} \cdot 2 \mathrm{H}_{2} \mathrm{O}$ in $100 \mathrm{ml}$ water.

Glycerol (99.5\% pure) was distilled twice at low pressure. The boiling point in the final distillation was $111^{\circ} \mathrm{C}$ at $0.01 \mathrm{mmHg}$.

DFx: after thawing the frozen DFx stock solution, the protein was stored at $4{ }^{\circ} \mathrm{C}$ as a mixture of grey and pink DFx in TRIS buffer $\mathrm{pH}$ 7.6. The DFx solution was filtered $(45 \mu \mathrm{m})$ before transfer to the buffer with no further purification. Transfer to other buffers was carried out by ultrafiltration. Grey DFx was prepared by adding a few granules of $\mathrm{K}_{2}\left[\mathrm{IrCl}_{6}\right]$ (potassium hexachloro-iridate(Iv), equilibrium oxidation potential $867 \mathrm{mV}$ versus $\mathrm{SHE}$ ) to the protein solution in an Amicon cell. Residual $\left[\mathrm{IrCl}_{6}\right]^{2-/ 3-}$ was removed by ultrafiltration. The final buffer (phosphate for all types of spectroscopy) was added and removed three times. NaCcd or CaCcd buffers was used for the low-temperature absorption spectroscopy experiments. Colourless DFx was prepared by adding a few granules of sodium dithionite to the Amicon cell. The solutions were ultrafiltered (YM3 filter) under argon. Colourless DFx is air-sensitive and oxidises partially to pink DFx if a reducing agent is not present. The final solutions were therefore always pink, i.e. they had become partially re-oxidised by air. A few granules of dithionite were added to the cuvette just before immersion into the cryostat. The absence of colour was verified by visual inspection. Pink DFx was prepared via complete reduction to the colourless form with dithionite, followed by removal of dithionite by ultrafiltration, and reoxidation of $\mathrm{Fe}_{\mathrm{I}}$ by air exposure. The cuvette with dithionite free DFx-solution was left overnight at $4{ }^{\circ} \mathrm{C}$ before use. The solutions became pink but the exact percentage of oxidation remained unknown.

\section{Absorption spectroscopy}

Low-temperature absorption spectra were recorded on a Varian Cary 5E UV-VIS-NIR double-beam spectrophotometer, equipped with an Oxford Instruments DN liquid bath cryostat. The cryostat was fitted into the sample beam with an in-house made adapter. The wavelength scale (reproducibility \pm 0.0125 $\mathrm{nm}$ or $1 / 2 \mathrm{~cm}^{-1}$ at $500 \mathrm{~nm}$ ) was calibrated against didymium and holmium oxide filter-glasses (Varian, Inc.). Pre-heating for at least two hours prior to use ensured stable conditions for spectrophotometer lamps (deuterium and tungsten type), the nitrogen purged optical system, and the detectors.

The cryostat sample chamber was modified by introduction of a helium gas purge facility to ensure atmospheric pressure conditions, effective thermal contact, and to prevent condensation of vaporised solvent at the inner optical windows. The optical window material (spectrosil-WF Quartz) is transparent in the range 190-3500 $\mathrm{nm}$.

Two computers controlled the spectrophotometer and the cryostat. One computer controlled a spectral scan every $25 \mathrm{~min}$ via the Varian software, the second one an Object Bench visual basic macro, which ensured sample temperature equilibrium in five $\min$ every $25 \mathrm{~min}$ ( $20 \mathrm{~min}$ was required for thermal equilibrium). Spectra were recorded at 45 different temperatures in steps of $5 \mathrm{~K}$ starting at $298 \mathrm{~K}$ and ending at $78 \mathrm{~K}$. The temperature sensors were calibrated at three points; against boiling liquid nitrogen, sublimating solid carbon dioxide, and 
an ice-water mixture. The absolute $T$-deviation was less than 1 $\mathrm{K}$, and the reproducibility better than $0.1 \mathrm{~K}$.

All samples were contained in polymethylmethacrylate (PMMA) cuvettes. PMMA has excellent transparency in the range from 260 to $1600 \mathrm{~nm}$. In contrast to glass or quartz, PMMA is robust to solidification of the buffer/glycerol mixture, but there is a significant spectral absorption difference within individual PMMA-cuvettes. The same cuvette was therefore used for both sample and background scanning. Absorption spectra in the $T$-range $298-78 \mathrm{~K}$ in the whole UV/VIS range for the three oxidation states of DFx in NaCcd buffer were first recorded. As DFx is engaged in $\mathrm{Ca}^{2+}$-dependent monomerdimer equilibria, similar recordings in $\mathrm{CaCcd}$ buffer were also undertaken. The RT equilibrium concentration of DFx, at which half of the monomer is left in a two-state monomerdimer model, is reported to be $1 \mu \mathrm{M} .{ }^{8}$ The DFx concentration in all samples was in the range $23-78 \mu \mathrm{M}$. This corresponds to monomer fractions in the range $15-8 \%$, if temperature, solvent, and other effects are disregarded, but these figures may vary significantly over the broad temperature range used.

\section{MCD and CD spectroscopy}

MCD spectroscopy was recorded with two spectropolarimeters. The first one was an in-house rebuilt Cary 14 spectrophotometer, the second one a Jasco-710 spectropolarimeter. Both instruments were equipped with the same Oxford Instruments SM3 liquid helium superconducting split-coil cryostat. The CD-intensity was calibrated against $5 \mathrm{~mm} 2\left\{\Lambda-(+)_{\mathrm{D}}-\left[\mathrm{Co}(\mathrm{en})_{3}\right]-\right.$ $\left.\mathrm{Cl}_{3}\right\} \cdot \mathrm{NaCl} \cdot 6 \mathrm{H}_{2} \mathrm{O}$ in water. ${ }^{22}$ The wavelength was calibrated with didymium and holmium oxide glass-filters.

The sample holder was fitted with a carbon-glass resistance temperature sensor and two wire-wound resistive heating elements with electrical supply from an ITC2 temperature controller. The best temperature control was achieved by running the ITC2 automatically with application of a slight liquid helium flow into the sample chamber while pumping to vacuum. The temperature was calibrated against boiling liquid helium and boiling nitrogen. The temperature range was $6.6-82 \mathrm{~K}$, and the magnetic field strength in the range $0-4 \mathrm{~T}$.

$90 \mu \mathrm{l}$ of DFx solution was mixed with $180 \mu$ glycerol in a round flat quartz QS $0.1 \mathrm{~cm}$ cuvette with a lower volume-limit of $283 \mu$ l. Mixing of sample and glycerol was continued until no refraction inhomogeneity could be observed. The cuvette was fastened in the sample holder and immersed into the cryostat while flushing with helium gas. The temperature was allowed to drop rapidly to $160 \mathrm{~K}$ before evacuation of the sample chamber. At $T=120 \mathrm{~K}$ the sample cracked into a fragmented but highly transparent glass. $T_{\mathrm{g}}$ of the aqueous/glycerol solvent mixture $1: 2(\mathrm{v} / \mathrm{v})$ is $\approx 163 \mathrm{~K}$. The transparency of the sample was controlled by visual inspection.

A CD spectrum was recorded before application of the magnetic field for all three DFx forms. After applying the field parallel to the direction of light propagation, MCD-spectra were recorded at $T=82,61.9,35.3,18.8,10.6,7.9$ and $6.6 \mathrm{~K}$ The magnetic field was then reversed and the spectra at 6.6 and $82 \mathrm{~K}$ recorded again. The corrected pure MCD and CD spectra were calculated as half of the differences and sums, respectively, of the parallel/antiparallel field spectral pairs.

\section{EPR spectroscopy}

A X-band EPR spectrum of grey DFx in glycerol/buffer $2: 1$ (v/v) at $T=9 \mathrm{~K}$ was recorded with a Bruker instrument (ESP 300 spectrometer, ER 083 Microwave Bridge, and ER 083 (200/60) magnet) combined with an Oxford Instruments liquid helium flow cryostat. The microwave frequency was $9.403 \mathrm{GHz}$ DFx was oxidized with $\mathrm{K}_{2}\left[\mathrm{IrCl}_{6}\right]$ and excess $\left[\mathrm{IrCl}_{6}\right]^{2-13-}$ removed by a three-fold solvent exchange in a Centricon ${ }^{\mathrm{TM}}$ concentration cell. Mixing of glycerol and DFx was carried out directly in the NMR-tube used for the EPR spectrometer. The final DFx concentration was $\approx 500 \mu \mathrm{M}$.

\section{Optical spectral analysis}

Optical spectral data were recorded as absorbance, $A$, versus wavelength, $\lambda(\mathrm{nm})$. Background-data were subtracted point by point with use of no interpolations. The spectra were corrected for nitrogen gas refraction and converted to $A$ versus wavenumber-in-vacuo, $\tilde{v} / \mu \mathrm{m}^{-1}$. The molar absorption coefficients, $\varepsilon$, and DFx-concentrations were determined via the RT $495 \mathrm{~nm}$ peak absorbance and molar absorption coefficient, $\varepsilon_{495}=4980$ $\mathrm{M}^{-1} \mathrm{~cm}^{-1}\left(498 \mathrm{~m}^{2} \mathrm{~mol}^{-1}\right){ }^{4}$ An overview of absorption-, MCDand CD- spectral observations of DFx at $T=78 \mathrm{~K}$ in the grey, pink and colourless forms is given in Table 1.

The files were transformed to molar-absorption-coefficient per wavenumber $\left(\varepsilon \tilde{v}^{-1} / 10^{-3} \mathrm{~m}^{3} \mathrm{~mol}^{-1}\right.$ versus $\left.\tilde{v} / \mu \mathrm{m}^{-1}\right)$ prior to bandshape analysis. Spectral data were analysed on the basis of optical bandshape theory for CT transitions in condensed media (see later). In the following we shall refer to spectral bands via their wavelengths at $T=78 \mathrm{~K}$. An overview of all fitted Gaussian bands is given in Tables 2 and 3.

\section{Results and discussion}

\section{General optical spectral properties of the three redox states of DFx}

Figs. 3-5 show VT absorption, MCD, and CD spectra of grey, pink and colourless DFx. Absorption spectra were recorded in the $T$-range 78 to $298 \mathrm{~K}$, VT-MCD and CD spectra in the range 6.6 to 82 K. Grey (Fig. 3) and pink DFx (Fig. 4) show strong visible $\left(\mathrm{Fe}^{3+} \longleftarrow\right.$ cys- $\left.\mathrm{S}^{-}\right) \mathrm{CT}$ absorption bands in the range 800 $\mathrm{nm}$ to $300 \mathrm{~nm}$ with maxima at 495 and $368 \mathrm{~nm}$ at RT. ${ }^{9}$ These peaks follow closely those of $D g$ Dx $(508$ and $372 \mathrm{~nm}),{ }^{23}$ Clostridium pasteurianum ( $C p)$ Dx (495 and $380 \mathrm{~nm})^{24}$ and $C p$ rubredoxin (Rd) (487 and $378 \mathrm{~nm}),{ }^{25}$ which can be regarded as reference molecules for $\mathrm{Fe}_{\mathrm{I}}$. Grey DFx exhibits additional broad RT contributions at 635 and $335 \mathrm{~nm} .{ }^{9}$ These can be assigned to $\left(\mathrm{Fe}^{3+} \leftarrow\right.$ cys- $\left.\mathrm{S}^{-}\right)$transitions in $\mathrm{Fe}_{\mathrm{II}}$ and have analogues in Archaeoglobus fulgidus (Af) Neelaredoxin (Nlr) (648 and $318 \mathrm{~nm}),{ }^{26}$ Treponema palladium $(T p) \mathrm{Nlr}(656 \text { and } 320 \mathrm{~nm})^{27}$ and $P f$ SOR $(635$ and $335 \mathrm{~nm}),{ }^{19}$ which constitute a second group of reference molecules. The absorption spectrum of colourless DFx (Fig. 5), shows little absorption in the VIS region, but several near-UV transitions of the $\left(\mathrm{Fe}^{2+} \longleftarrow\right.$ cys- $\left.\mathrm{S}^{-}\right)$ type between 380 and $280 \mathrm{~nm}$. This picture has been confirmed by recombinant techniques where the $D v$ DFx domains I and II have been expressed separately and characterised optically and in other ways. ${ }^{17}$

Much more detail is disclosed at low temperature and in combination with VT-MCD and CD. Common to the UV/VIS spectra in Figs. 3-5 is a clear increase in band resolution and a weak shift in band position as the temperature is lowered. We refer to band positions particularly at $T=78 \mathrm{~K}$ in the following.

Peaks at $440 \mathrm{~nm}$ and $565 \mathrm{~nm}$ in the grey DFx spectrum, largely hidden at RT emerge. These transitions are not apparent in pink $\mathrm{DFx}$ and can be associated with $\mathrm{Fe}_{\mathrm{I}}$. The spectral features of pink DFx are less resolved at low temperature, perhaps in part due to difficulties in controlling the redox level of this form. The VT MCD and CD spectra are, however, much better resolved. The spectral detail in Fig. 3 warrants bandshape analysis of grey DFx. A feature of several of the bands is a transition from almost temperature independent bandwidth at low temperatures to approximately $\sqrt{ } T$-dependence at higher temperature. This could reflect a change in the mode of nuclear displacement that accompanies the transitions, from thermal motion at high temperatures to nuclear tunnelling at low temperatures. ${ }^{2}$ Several of the apparent coupling factors are, however, far too large and inhomogeneous broadening or conformational inhomogeneity must also be invoked. None 
Table 1 Spectral characteristics (UV/VIS absorption, MCD and CD) of grey, pink, and colourless (Col) DFx

\begin{tabular}{|c|c|c|c|c|c|c|c|c|c|c|}
\hline \multirow[b]{3}{*}{ Wavelength/nm } & \multicolumn{3}{|c|}{ Absorption } & \multicolumn{3}{|l|}{ MCD } & \multicolumn{3}{|l|}{$\mathrm{CD}$} & \multirow[b]{3}{*}{ Assignment } \\
\hline & \multicolumn{3}{|c|}{ Wavenumber/ $/ \mu \mathrm{m}^{-1}$} & \multicolumn{3}{|c|}{ Wavenumber $/ \mu \mathrm{m}^{-1}$} & \multicolumn{3}{|c|}{ Wavenumber $/ \mu \mathrm{m}^{-1}$} & \\
\hline & Grey & Pink & Col & Grey & Pink & $\mathrm{Col}$ & Grey & Pink & $\mathrm{Col}$ & \\
\hline 280 & 3.57 & 3.57 & 3.57 & & & & & & & $\pi^{*} \longleftarrow \pi$ \\
\hline 299 & $3.35 \mathrm{sh}$ & $3.35 \mathrm{sh}$ & & & & & & & & ${ }^{\mathrm{Na}} \mathrm{G}_{0},{ }^{\mathrm{Ca}} \mathrm{G}_{0}$ \\
\hline 304 & & & $3.30 \mathrm{sh}$ & & & $3.29-$ & $3.30+$ & $3.30+$ & & redI \\
\hline 312 & & & $3.21 \mathrm{sh}$ & & $3.18-$ & $A-$ & & & & redII, ${ }^{\mathrm{Ca}} \mathrm{G}_{1}$ \\
\hline 319 & 3.13 & $3.14 \mathrm{sh}$ & & $3.15+$ & & $3.13+$ & & $3.15-$ & $3.15+$ & oxII, ${ }^{\mathrm{Na}} \mathrm{G}_{1}$ \\
\hline 326 & & & & & & & $3.07-$ & & & \\
\hline 335 & & 2.94 & $2.95 \mathrm{sh}$ & $2.98-$ & $2.98+$ & $2.95 \mathrm{sh}$ & & $2.98+$ & $2.95+$ & oxII \\
\hline 364 & 2.75 & & & & & & $2.74+$ & $2.72-$ & & oxI, oxII, ${ }^{\mathrm{Na}} \mathrm{G}_{2}$ \\
\hline 370 & & $2.70 \mathrm{sh}$ & $2.70 \mathrm{sh}$ & $2.70+$ & & $2.70 \mathrm{sh}$ & & & & ${ }^{\mathrm{Ca}} \mathrm{G}_{2}$ \\
\hline 385 & & & & & & & & & $2.60+$ & \\
\hline 392 & & & & & $2.55 \mathrm{sh}-$ & & $2.55-$ & & & \\
\hline 408 & & & & & & & $2.46+$ & $2.45+$ & & oxI, ${ }^{\mathrm{Na}} \mathrm{G}_{3},{ }^{\mathrm{Ca}} \mathrm{G}_{3}$ \\
\hline 417 & & & & $2.40-$ & $2.40-$ & & & & & \\
\hline 426 & & $2.35 \mathrm{sh}$ & & & $2.35 \mathrm{sh}-$ & & $2.35-$ & & & \\
\hline 440 & 2.27 & & & & & & $2.26+$ & $2.28-$ & & oxI, ${ }^{\mathrm{Na}} \mathrm{G}_{4},{ }^{\mathrm{Ca}} \mathrm{G}_{4}$ \\
\hline 461 & & & & $2.17-$ & & & & & & \\
\hline 476 & & & & & $2.10 \mathrm{sh}-$ & & & $2.10 \mathrm{sh}-$ & & \\
\hline 490 & & 2.04 & & & & & & & & \\
\hline 500 & 2.00 & & & $\mathrm{~A}-$ & & & $2.00-$ & & & oxI, ${ }^{\mathrm{Na}} \mathrm{G}_{5},{ }^{\mathrm{Ca}} \mathrm{G}_{5}$ \\
\hline 521 & & & & $1.92+$ & & & & $1.93+$ & & \\
\hline 543 & & $1.85 \mathrm{sh}$ & & & $1.83+$ & & & & & oxII, ${ }^{\mathrm{Ca}} \mathrm{G}_{6}$ \\
\hline 565 & 1.77 & & & $1.78+$ & & & $1.77+$ & $1.78 \mathrm{sh}-$ & & oxI, ${ }^{\mathrm{Na}} \mathrm{G}_{6},{ }^{\mathrm{Ca}} \mathrm{G}_{7}$ \\
\hline 610 & & & & & & & $1.64-$ & & & oxII, ${ }^{\mathrm{Na}} \mathrm{G}_{7}$ \\
\hline 625 & & $1.60 \mathrm{sh}$ & & & & & & $1.60-$ & & ${ }^{\mathrm{Ca}} \mathrm{G}_{8}$ \\
\hline 641 & $1.56 \mathrm{sh}$ & & & $1.56 \mathrm{sh}+$ & & & & & & oxII, ${ }^{\mathrm{Na}} \mathrm{G}_{8}$ \\
\hline 654 & & & & & $1.53 \mathrm{sh}+$ & & & & & \\
\hline 694 & & $1.45 \mathrm{sh}$ & & & & & $1.44+$ & & & \\
\hline 714 & $1.40 \mathrm{sh}$ & & & $1.40-$ & & & & & & $(d \longleftarrow d)$ \\
\hline 769 & & & & $1.30+$ & & & & & & \\
\hline 833 & $1.20 \mathrm{sh}$ & & & & & & & & & \\
\hline
\end{tabular}

Wavelengths $(\mathrm{nm}$ at $78 \mathrm{~K})$ are listed to the left. Wavenumbers $\left(\mu \mathrm{m}^{-1}\right)$ for absorption maxima and local maxima or minima in $\mathrm{MCD}$ and $\mathrm{CD}$ are shown in the middle part. "sh" indicates a shoulder and "+" or "-" indicate local MCD or CD extrema. These are in MCD mostly due to C-terms but an "A" indicates the presence of an A-term.

Table 2 Gaussian bandshape parameters of the UV/VIS absorption spectra of grey DFx

\begin{tabular}{|c|c|c|c|c|c|c|c|c|c|}
\hline \multirow{3}{*}{$\frac{\text { Band }}{\mathrm{G}_{i}}$} & \multirow{2}{*}{\multicolumn{2}{|c|}{$\begin{array}{l}\text { Position/energy } \\
T=78 \mathrm{~K}\end{array}$}} & \multirow{3}{*}{$\begin{array}{l}\text { Integrated } \\
\text { intensity } \\
T=78 \mathrm{~K} \\
\Gamma_{i} / \mathrm{m}^{2} \mathrm{~mol}^{-1}\end{array}$} & \multicolumn{3}{|c|}{ Gaussian widths (FWHM) } & \multirow{3}{*}{$\begin{array}{l}\text { Local mode } \\
\text { frequency } \\
T=78 \mathrm{~K} \\
\frac{T \omega_{\mathrm{m}} / \mathrm{cm}^{-1}}{}\end{array}$} & \multirow{3}{*}{$\begin{array}{l}\text { Reorganisation } \\
\text { energy } \\
T=298 \mathrm{~K} \\
E_{\mathrm{r}} / \mathrm{cm}^{-1}\end{array}$} & \multirow[b]{3}{*}{ Assignment } \\
\hline & & & & \multirow{2}{*}{$\frac{T=78 \mathrm{~K}}{\Delta_{i} / \mathrm{cm}^{-1}}$} & \multirow{2}{*}{$\frac{T=188 \mathrm{~K}}{\Delta_{i} / \mathrm{cm}^{-1}}$} & \multirow{2}{*}{$\frac{T=298 \mathrm{~K}}{\Delta_{i} / \mathrm{cm}^{-1}}$} & & & \\
\hline & $\lambda_{i} / \mathrm{nm}$ & $v_{i} / \mu \mathrm{m}^{-1}$ & & & & & & & \\
\hline$\left(\mathrm{G}_{0}\right)$ & 296 & 3.38 & $31.6 \pm 1.0$ & $1350 \pm 21$ & $1618 \pm 21$ & $2134 \pm 22$ & $165 \pm 3$ & $2000 \pm 21$ & $\left(\pi^{*} \longleftarrow \pi\right)$ \\
\hline $\mathrm{G}_{1}$ & 318 & 3.15 & $121.1 \pm 1.4$ & $3618 \pm 26$ & $3666 \pm 24$ & $3679 \pm 21$ & $400 \pm 3$ & $5900 \pm 34$ & II \\
\hline $\mathrm{G}_{2}$ & 366 & 2.73 & $161.9 \pm 1.8$ & $4367 \pm 41$ & $4629 \pm 35$ & $4957 \pm 31$ & $320 \pm 3$ & $10700 \pm 67$ & $\mathrm{I}+\mathrm{II}$ \\
\hline $\mathrm{G}_{3}$ & 409 & 2.44 & $4.4 \pm 1.4$ & $1597 \pm 116$ & $1715 \pm 96$ & $1875 \pm 82$ & $300 \pm 22$ & $1530 \pm 67$ & I \\
\hline $\mathrm{G}_{4}$ & 442 & 2.26 & $65.3 \pm 4.6$ & $3100 \pm 162$ & $2998 \pm 165$ & $3015 \pm 168$ & $440 \pm 23$ & $3950 \pm 220$ & $\mathrm{I}$ \\
\hline $\mathrm{G}_{5}$ & 507 & 1.97 & $65.4 \pm 3.5$ & $2750 \pm 79$ & $3328 \pm 151$ & $4493 \pm 258$ & $150 \pm 4$ & $8800 \pm 505$ & I \\
\hline $\mathrm{G}_{6}$ & 567 & 1.76 & $16.3 \pm 5.9$ & $1479 \pm 82$ & $1604 \pm 128$ & $1588 \pm 244$ & $355 \pm 20$ & $1100 \pm 169$ & $\mathrm{I}+\mathrm{II}$ \\
\hline $\mathrm{G}_{7}$ & 608 & 1.64 & $24.3 \pm 17.8$ & $2273 \pm 732$ & $2269 \pm 674$ & $2124 \pm 569$ & $440 \pm 142$ & $2200 \pm 589$ & II \\
\hline $\mathrm{G}_{8}$ & 677 & 1.48 & $25.8 \pm 11.2$ & $2465 \pm 234$ & $2739 \pm 208$ & $3813 \pm 135$ & $170 \pm 16$ & $6530 \pm 223$ & II \\
\hline
\end{tabular}

In sodium cacodylate buffer/glycerol, $1: 2(\mathrm{v} / \mathrm{v})\left(I=30 \mathrm{~mm}, \mathrm{pH}=7.0\right.$ before addition of glycerol) Fig. $6 \mathrm{a}$. The eight $\mathrm{Gaussian} \mathrm{CT}$ bands, $\mathrm{G}_{i}, i=1-8$ are characterized by their maximum wavelength, $\lambda_{i}$, and frequency, $v_{i}$, integrated band intensities, $\Gamma_{i}$, and bandwidths (FWHM), $\Delta_{i}$, at the three temperatures indicated. The average vibrational frequency, $\omega_{\mathrm{m}}$ is determined by the temperature variation of the bandwidths (Fig. $9 \mathrm{a}$ ). The total reorganization free energy, $E_{\mathrm{r}}$, is obtained from the high-temperature form of the bandwidth in eqn. $(7 \mathrm{a})$ for $\omega_{\mathrm{m}} \geq 300 \mathrm{~cm}^{-1}$. The $T$-independent bands $\left(\mathrm{G}_{1}, \mathrm{G}_{4}\right.$, and $\left.\mathrm{G}_{7}\right)$ can only provide one form, eqn. (7b).

of the CT spectra show vibrational fine-structure which is, however, visible at low $T$ in the strong $280 \mathrm{~nm}$ aromatic protein region (DFx contains 3 Trp, 3 Phe, 4 Tyr, and 9 His).

The VT-MCD spectra follow the low- $T$ absorption spectra but also show differences, both from the absorption spectra and from the spectra of monomeric reference molecules $D g \mathrm{Dx},{ }^{23}$ Af $\mathrm{Nlr}^{22,27}$ and $P f \mathrm{SOR}^{19}$ The $641 \mathrm{~nm}$ and $335 \mathrm{~nm}$ contributions of grey $\mathrm{DFx}$ assigned to $\mathrm{Fe}_{\mathrm{II}}$ correspond to positive C-terms in the VT-MCD spectrum (Fig. 3). Other strong positive C-terms are located at $565 \mathrm{~nm}, 521 \mathrm{~nm}$ and $370 \mathrm{~nm}$. The
$565 \mathrm{~nm}$ and $370 \mathrm{~nm}$ C-terms accord with the absorption bands for $\mathrm{Fe}_{\mathrm{I}}$ at $565 \mathrm{~nm}$ and $364 \mathrm{~nm}$, which become resolved at low temperatures. The $565 \mathrm{~nm}$ band is also likely to hold a $\mathrm{Fe}_{\text {II }}$ component, by comparison with the VT-MCD spectra of $P f$ SOR $^{19}$ and with pink DFx (Fig. 4). The MCD band around 521 nm holds a $T$-dependent A-term as well. This transition accords with the $500 \mathrm{~nm} \mathrm{Fe}$ absorption band disclosed at low temperatures and overlapping with the $440 \mathrm{~nm}$ band. The 521 and 440 $\mathrm{nm} \mathrm{Fe}$ I absorption bands are thus convoluted into the $495 \mathrm{~nm}$ band at RT but clearly resolved both in the low-temperature 
Table 3 Gaussian bandshape parameters of the UV/VIS absorption spectra of grey DFx

\begin{tabular}{|c|c|c|c|c|c|c|c|c|c|}
\hline \multirow{3}{*}{$\frac{\text { Band }}{\mathrm{G}_{i}}$} & \multirow{2}{*}{\multicolumn{2}{|c|}{$\begin{array}{l}\text { Position/energy } \\
T=78 \mathrm{~K}\end{array}$}} & \multirow{3}{*}{$\begin{array}{l}\text { Integrated } \\
\text { intensity } \\
T=78 \mathrm{~K} \\
\Gamma_{i} / \mathrm{m}^{2} \mathrm{~mol}^{-1}\end{array}$} & \multicolumn{3}{|c|}{ Gaussian widths (FWHM) } & \multirow{3}{*}{$\begin{array}{l}\text { Local mode } \\
\text { frequency } \\
T=78 \mathrm{~K} \\
\frac{\hbar \omega_{\mathrm{m}} / \mathrm{cm}^{-1}}{}\end{array}$} & \multirow{3}{*}{$\begin{array}{l}\text { Reorganisation } \\
\text { energy } \\
T=298 \mathrm{~K} \\
E_{\mathrm{r}} / \mathrm{cm}^{-1}\end{array}$} & \multirow[b]{3}{*}{ Assignment } \\
\hline & & & & \multirow{2}{*}{$\frac{T=78 \mathrm{~K}}{\Delta v / \mathrm{cm}^{-1}}$} & \multirow{2}{*}{$\frac{T=188 \mathrm{~K}}{\Delta_{i} / \mathrm{cm}^{-1}}$} & \multirow{2}{*}{$\frac{T=298 \mathrm{~K}}{\Delta_{i} / \mathrm{cm}^{-1}}$} & & & \\
\hline & $\lambda_{i} / \mathrm{nm}$ & $v_{i} / \mu \mathrm{m}^{-1}$ & & & & & & & \\
\hline$\left(\mathrm{G}_{0}\right)$ & 295 & 3.38 & $30.8 \pm 0.9$ & $1366 \pm 16$ & $1691 \pm 23$ & $2322 \pm 37$ & $143 \pm 2$ & $2350 \pm 37$ & $\left(\pi^{*} \longleftarrow \pi\right)$ \\
\hline $\mathrm{G}_{1}$ & 313 & 3.20 & $168.2 \pm 3.5$ & $5469 \pm 95$ & $5136 \pm 118$ & $4204 \pm 87$ & $700 \pm 12$ & $7700 \pm 159$ & II \\
\hline $\mathrm{G}_{2}$ & 370 & 2.70 & $107.9 \pm 2.5$ & $4553 \pm 54$ & $5039 \pm 79$ & $5547 \pm 188$ & $279 \pm 3$ & $13400 \pm 454$ & $\mathrm{I}+\mathrm{II}$ \\
\hline $\mathrm{G}_{3}$ & 409 & 2.44 & $4.2 \pm 1.2$ & $1745 \pm 101$ & $1805 \pm 107$ & $1924 \pm 147$ & $340 \pm 20$ & $1615 \pm 123$ & I \\
\hline $\mathrm{G}_{4}$ & 440 & 2.27 & $38.2 \pm 3.7$ & $2892 \pm 159$ & $2759 \pm 183$ & $2838 \pm 500$ & $430 \pm 24$ & $3510 \pm 618$ & I \\
\hline $\mathrm{G}_{5}$ & 503 & 1.99 & $49.5 \pm 2.7$ & $3200 \pm 105$ & $4166 \pm 233$ & $5041 \pm 1229$ & $167 \pm 5$ & $11060 \pm 2696$ & I \\
\hline $\mathrm{G}_{6}$ & 558 & 1.79 & $1.7 \pm 3.6$ & $1082 \pm 268$ & $1466 \pm 370$ & $1287 \pm 209$ & $293 \pm 73$ & $720 \pm 117$ & $\mathrm{I}+\mathrm{II}$ \\
\hline $\mathrm{G}_{7}$ & 576 & 1.74 & $4.5 \pm 3.9$ & $1377 \pm 280$ & $1882 \pm 1597$ & $2393 \pm 287$ & $137 \pm 28$ & $2495 \pm 299$ & $\mathrm{I}+\mathrm{II}$ \\
\hline $\mathrm{G}_{8}$ & 635 & 1.57 & $45.6 \pm 0.5$ & $4169 \pm 30$ & $4154 \pm 139$ & $4620 \pm 238$ & $337 \pm 2$ & $9300 \pm 479$ & II \\
\hline
\end{tabular}

In calcium cacodylate buffer/glycerol, $1: 2(\mathrm{v} / \mathrm{v})(I=30 \mathrm{~mm}, \mathrm{pH}=7.0$ before addition of glycerol $)$ Fig. $6 \mathrm{~b}$. Same notation and procedure as in Table 2 .

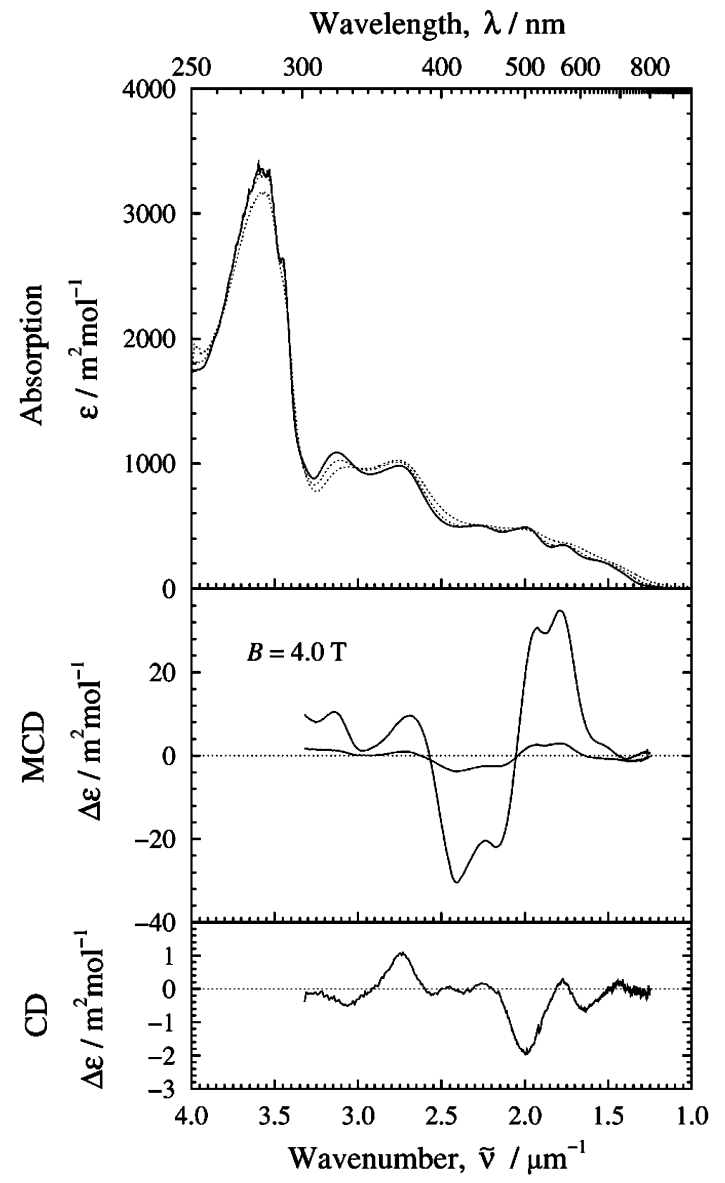

Fig. 3 Spectra of fully oxidised, grey Desulfovibrio desulfuricans DFx. Top scale: wavelength $\lambda / \mathrm{nm}$. Left scales: molar absorption coefficien and the corresponding difference; $\varepsilon / \mathrm{m}^{2} \mathrm{~mol}^{-1}$ and $\Delta \varepsilon / \mathrm{m}^{2} \mathrm{~mol}^{-1}$ respectively. Bottom scale: in-vacuo wavenumber $\tilde{v} / \mu \mathrm{m}^{-1}$. Upper panel absorption spectra at $78 \mathrm{~K}(-)$; spectra at 188 and $298 \mathrm{~K}$ are also shown (. . .). Sodium cacodylate buffer $\mathrm{pH} 7.0, I=30 \mathrm{~mm}$ mixed with glycerol, in the ratio $1: 2(\mathrm{v} / \mathrm{v})$. Middle panel: MCD spectra at $T=80 \mathrm{~K}$ (small intensity -), and 6.6 K (large intensity -). Lower panel: CD spectrum at $T=6.6 \mathrm{~K}(-)$. The buffer for the MCD and CD spectra was sodium phosphate $\mathrm{pH} 7.0, I=30 \mathrm{~mm}$ mixed with glycerol, $1: 2(\mathrm{v} / \mathrm{v})$.

absorption spectra and in the VT-MCD spectra at $500 \mathrm{~nm}$. Two strong negative MCD features at 417 and $461 \mathrm{~nm}$ are likely to accord with two components of the $440 \mathrm{~nm}$ absorption band.

In summary, grey DFx displays two broad absorption bands at $500 \mathrm{~nm}(\mathrm{RT} 495 \mathrm{~nm})$ and $370 \mathrm{~nm}$ (RT $368 \mathrm{~nm}$ ) associated with $\mathrm{Fe}_{\mathrm{I}}$, and two other absorption contributions at $641 \mathrm{~nm}$ (RT $635 \mathrm{~nm}$ ) and $335 \mathrm{~nm}$ (RT $335 \mathrm{~nm}$ ) which also appear directly as MCD bands, associated with $\mathrm{Fe}_{\mathrm{II}}$. The $500 \mathrm{~nm}$ absorption band is resolved into three bands at 440 and $500 \mathrm{~nm}$ at low temperatures where the additional band at $565 \mathrm{~nm}$ is also disclosed.

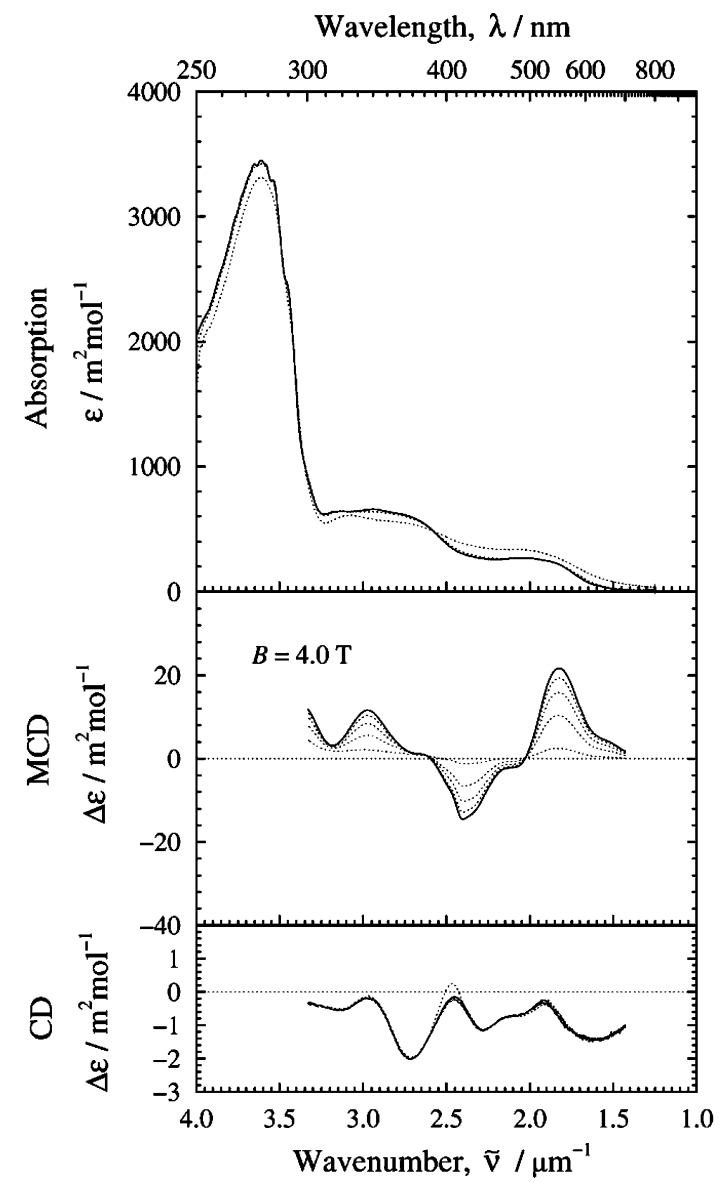

Fig. 4 Spectra of half-reduced, pink Desulfovibrio desulfuricans DFx. Top scale: wavelength $\lambda / \mathrm{nm}$. Left scales: molar absorption coefficient and the corresponding difference; $\varepsilon / \mathrm{m}^{2} \mathrm{~mol}^{-1}$ and $\Delta \varepsilon / \mathrm{m}^{2} \mathrm{~mol}^{-1}$, respectively. Bottom scale: in-vacuo wavenumber $\tilde{v} / \mu \mathrm{m}^{-1}$. Upper panel:

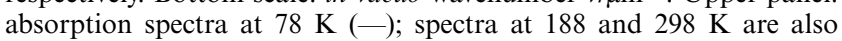
shown (. . .). Sodium cacodylate buffer $\mathrm{pH} 7.0, I=30 \mathrm{~mm}$ mixed with glycerol, $1: 2(\mathrm{v} / \mathrm{v})$. Middle panel: MCD spectra with increasing peak height at $T=80 \mathrm{~K}(\ldots), 16.6 \mathrm{~K}(\ldots), 10.2 \mathrm{~K}(\ldots), 7.9 \mathrm{~K}(\ldots)$, and $6.6 \mathrm{~K}$ (-). Lower panel: CD spectrum at $T=80 \mathrm{~K}(\ldots), 16.6 \mathrm{~K}(\ldots), 10.2 \mathrm{~K}$ $(\ldots), 7.9 \mathrm{~K}(\ldots)$, and $6.6 \mathrm{~K}(-)$. The buffer for the MCD and CD spectra was sodium phosphate $\mathrm{pH} 7.0, I=30 \mathrm{~mm}$ mixed with glycerol, $1: 2(\mathrm{v} / \mathrm{v})$.

The sub-band at $440 \mathrm{~nm}$ of the $500 \mathrm{~nm} \mathrm{Fe}$ band is further composite as seen by a negative C-term VT-MCD band at $417 \mathrm{~nm}$ and a negative A-term band at $500 \mathrm{~nm}$ but VT-MCD contributions from $\mathrm{Fe}_{\text {II }}$ may also contribute here. The 335 and $641 \mathrm{~nm} \mathrm{Fe}_{\mathrm{II}}$ assigned absorption contributions remain largely unresolved in both absorption and VT-MCD spectroscopy (see, however, later and Table 2) but the strong A-term MCD feature at $500 \mathrm{~nm}$ and C-term feature at $565 \mathrm{~nm}$ are likely to contain contributions from both centres. 


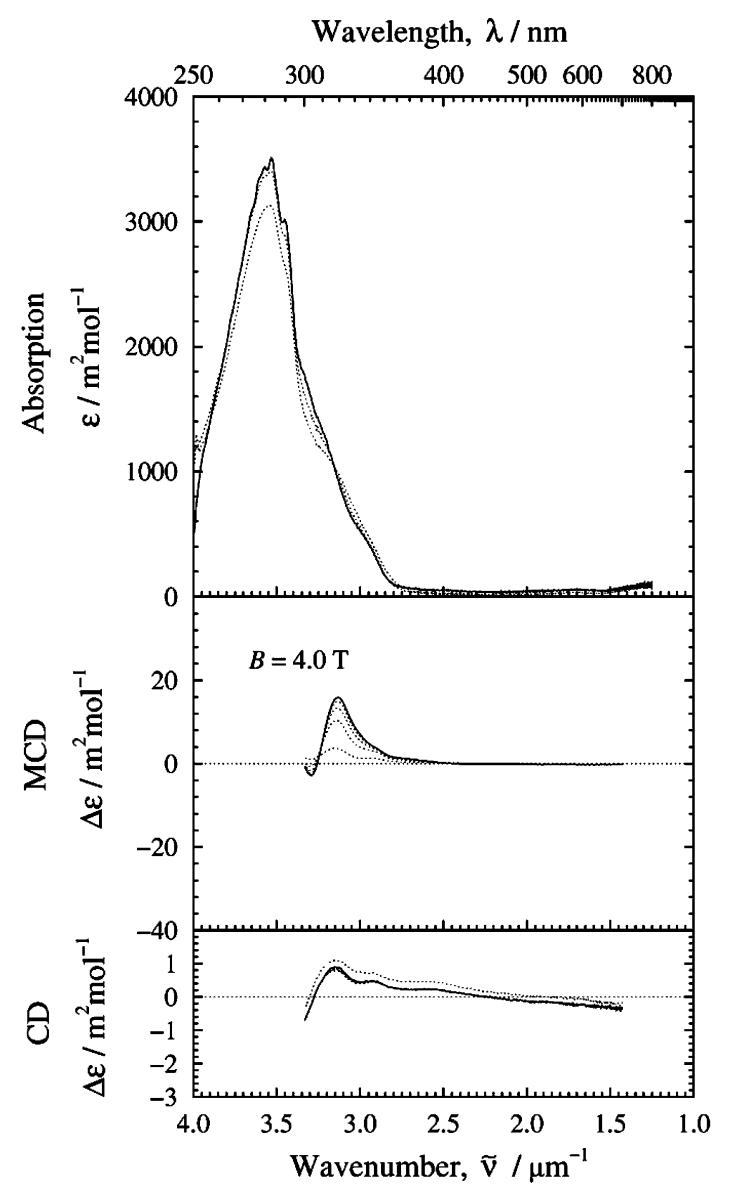

Fig. 5 Spectra of fully reduced, colourless Desulfovibrio desulfurican DFx. Upper panel: wavelength $\lambda / \mathrm{nm}$. Left scales: molar absorption coefficient and the corresponding difference; $\varepsilon / \mathrm{m}^{2} \mathrm{~mol}^{-1}$ and $\Delta \varepsilon / \mathrm{m}^{2}$ $\mathrm{mol}^{-1}$, respectively. Bottom scale: in-vacuo wavenumber $\tilde{v} / \mu \mathrm{m}^{-1}$. Sodium dithionite background spectra have been subtracted. Upper panel: absorption spectra at $78 \mathrm{~K}(-)$; spectra at 188 and $298 \mathrm{~K}$ are also shown (. . .). Sodium cacodylate buffer $\mathrm{pH} 7.0, I=30 \mathrm{~mm}$ mixed with glycerol in the ratio $1: 2(\mathrm{v} / \mathrm{v})$. Middle panel: MCD spectra with increasing peak height at $T=80 \mathrm{~K}(\ldots), 16.6 \mathrm{~K}(\ldots), 10.2 \mathrm{~K}(\ldots), 7.9 \mathrm{~K}$ $(\ldots)$, and $6.6 \mathrm{~K}(-)$. Lower panel: $\mathrm{CD}$ spectrum with increasing peak height at $T=80 \mathrm{~K}(\ldots), 16.6 \mathrm{~K}(\ldots), 10.2 \mathrm{~K}(\ldots), 7.9 \mathrm{~K}(\ldots)$, and $6.6 \mathrm{~K}$ (-). The buffer for the MCD and CD spectra was sodium phosphate $\mathrm{pH} 7.0, I=30 \mathrm{~mm}$ mixed with glycerol, $1: 2(\mathrm{v} / \mathrm{v})$.

The absorption bands of pink DFx, Fig. 4, are less well resolved than for grey DFx, with little improved resolution at low temperatures. As noted, both the absorption spectra and the VT-MCD spectra may contain contributions from grey DFx. Bandshape analysis is therefore focused on grey DFx but the absorption and VT-MCD spectra of pink DFx offer other observations. Broad absorption bands at 490 to $543 \mathrm{~nm}, 370$, 335 and $319 \mathrm{~nm}$ can be identified. The former two bands are associated with oxidised $\mathrm{Fe}_{\mathrm{I}}$ but clearly broadened and red-shifted compared with grey DFx. The $319 \mathrm{~nm}$ band is likely to hold components from both oxidised $\mathrm{Fe}_{\mathrm{I}}$ and reduced $\mathrm{Fe}_{\mathrm{II}}$. The strong VT-MCD features in the 520 to $560 \mathrm{~nm}$ and 400 to $500 \mathrm{~nm}$ regions, Fig. 4, accord with the absorption spectra and are dominated by contributions from oxidised $\mathrm{Fe}_{\mathrm{I}}$, as colourless DFx only displays VT-MCD in the 310 to $340 \mathrm{~nm}$ region (Fig. 5, cf. below). The A-term VT-MCD transition of oxidised $\mathrm{Fe}_{\mathrm{I}}$ has, interestingly, disappeared but a significant negative $\mathrm{C}$-term at $\approx 476 \mathrm{~nm}$ remains. Both a $\mathrm{Fe}_{\mathrm{II}} \mathrm{A}$-term and a $\mathrm{Fe}_{\mathrm{I}}$ C-term therefore contribute to the strong VT-MCD feature at $\approx 500 \mathrm{~nm}$ in grey DFx. The VT-MCD band intensities are, however, significantly reduced compared with the similar bands of grey DFx and less resolved than the corresponding VTMCD bands for oxidised $C p \mathrm{Rd}$ and $D g$ Dx. ${ }^{24,27}$ The former effect substantiates that oxidised $\mathrm{Fe}_{\mathrm{II}}$ contributes significantly to the VT-MCD bands in the whole 450 to $641 \mathrm{~nm}$ range in grey
DFx, as anticipated from the VT-MCD spectra of oxidised Pf SOR where a positive MCD-feature emerges at $526 \mathrm{~nm} .{ }^{19}$ The $370 \mathrm{~nm} \mathrm{Fe}$ I VT-MCD feature in grey DFx is significantly blue-shifted to $335 \mathrm{~nm}$ in pink DFx. Together with the drastically changed character of the negative 417 and $476 \mathrm{~nm}$ VT-MCD features between grey and pink DFx, the notable absorption band shifts are indications that major conformational reorganisation extending to the whole region between the centres, or temperature dependent monomer-dimer equilibrium shifts accompany reduction of $\mathrm{Fe}_{\mathrm{II}}$. Major conformational reorganisation also emerges from the absorption spectral analysis in the next section.

Colourless DFx has absorption shoulders at 304, 312 and 335 $\mathrm{nm}$, most likely associated with $\left(\mathrm{Fe}^{2+} \longleftarrow \mathrm{S}^{-}\right)$transitions. The first and last can be assigned to $\mathrm{Fe}_{\mathrm{I}}$, the middle to $\mathrm{Fe}_{\mathrm{II}}$. This is matched by the VT-MCD spectrum, which shows a single strong positive feature at $319 \mathrm{~nm}$ with a shoulder at $335 \mathrm{~nm}$ and a moderate negative feature at $304 \mathrm{~nm}$ (Fig. 5). Dithionite has a MCD band in the same region but much weaker than those in Fig. 5. Three MCD features of colourless DFx (304 nm -, $319 \mathrm{~nm}+$ and $335 \mathrm{~nm}+$ ) match similar bands of reduced $P f$ SOR $(280 \mathrm{~nm}-, 295 \mathrm{~nm}+$ and $321 \mathrm{~nm}+){ }^{19}$ but are red-shifted compared with the $P f$ SOR bands. The different Fe-ligand distances in $P f \mathrm{SOR}^{28}$ (here the Cys-S corresponding to Cys 115 is significantly more distant while the His corresponding to His 48 and His 74 are closer and His68 and His118 are more distant) could explain both the different energies and intensity ratios. The latter may also be indicative of a component from $\mathrm{Fe}_{\mathrm{I}}$ such as expected from a similar VT-MCD band of reduced $D g$ Rd $(306 \mathrm{~nm}-$ and $333 \mathrm{~nm}+) .{ }^{29}$ The band shift again suggests that other conformational changes accompany the conversion of pink to colourless DFx.

Fig. 6a and $\mathrm{b}$ show the UV/VIS absorption spectra of grey DFx, in $\varepsilon \tilde{v}^{-1} / 10^{-3} \mathrm{~m}^{3} \mathrm{~mol}^{-1}$, versus $\tilde{v} / \mu \mathrm{m}^{-1}$ form, in $\mathrm{NaCcd}$ and CaCcd buffers, respectively, at $T=78,188$, and $298 \mathrm{~K}$. The dimeric form of DFx is likely to be promoted in CaCcd buffer. The CT transitions are broadly the same as in $\mathrm{NaCcd}$ buffer but changes in width and maximum of some bands are conspicuous in the analysis below. This also applies to pink DFx (not shown).

\section{EPR spectroscopy}

An EPR-spectrum of grey DFx was also recorded, Fig. 7, upper panel. It consists of contributions from both centres. The resonances at $g_{\text {eff }}=7.88,5.8, \approx 4$ (clearly of composite nature) and 1.8 are also found in pink DFx and are caused by oxidised $\mathrm{Fe}_{\mathrm{I}}{ }^{6}{ }^{6}$ The resonance at $g_{\text {eff }}=9.11$ and further contributions to the resonance at $\approx 4.3$ are observed only in grey $\mathrm{DFx}$ and accordingly, assigned to oxidised $\mathrm{Fe}_{\mathrm{II}} \cdot{ }^{11}$ Later work has confirmed this assignment. ${ }^{17}$

The simulated EPR-spectra are shown in Fig. 7, lower panel, which shows the generated spectra of the two oxidised centres separately and their sum. Each spectrum was generated assuming a ${ }^{6} \mathrm{~A}$ ground state of a $\mathrm{d}^{5}$ electron system using the spin-Hamiltonian

$$
\mathscr{H}_{\text {spin }}=\mu_{\mathrm{B}} g_{\mathrm{e}} \boldsymbol{B} \cdot \hat{\boldsymbol{S}}+\mathscr{H}_{\text {zfs }}
$$

where $\mu_{\mathrm{B}}$ is the Bohr magneton, $g_{\mathrm{e}}$ the Lande $g$-factor for the free electron, $\boldsymbol{B}$ the applied external magnetic field, $\hat{\boldsymbol{S}}$ the total spin angular momentum operator and $\mathscr{H}_{\mathbf{z f s}}$ the zero-field splitting Hamiltonian. The latter comprises here only quadratic terms

$$
\mathscr{H}_{\text {zfs }}=D\left(\hat{S}_{z}^{2}-\frac{1}{3} \hat{\boldsymbol{S}}^{2}\right)+E\left(\hat{S}_{x}^{2}+\hat{S}_{y}^{2}\right)
$$

where $D$ and $E$ are the zero-field splitting parameters. It follows from early work ${ }^{30}$ that a $E / D$ ratio can always be chosen within the interval $-1 / 3<E / D<+1 / 3$ with $E$ positive and $D$ either positive or negative. Further, close to the limiting cases $E / D=$ 

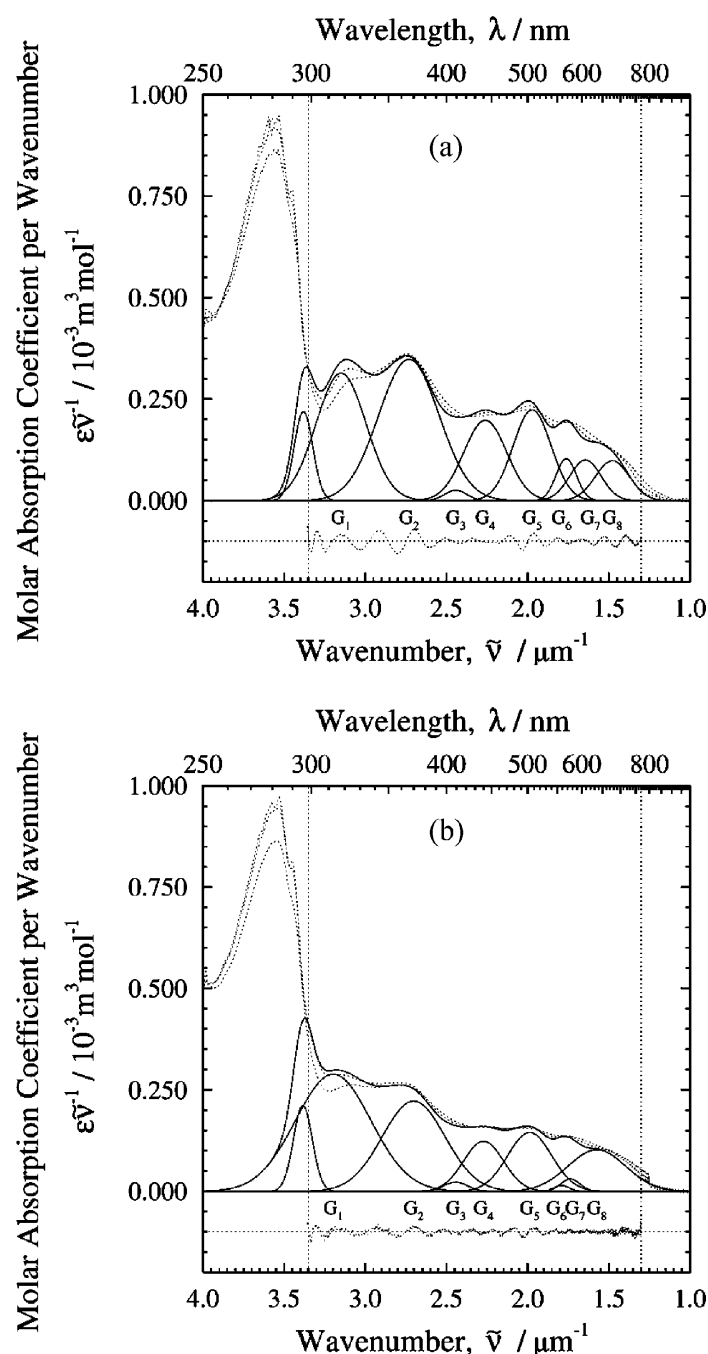

Fig. 6 Top scale: wavelength $\lambda / \mathrm{nm}$. Left scale: molar absorption coefficient per wavenumber $\varepsilon \tilde{v}^{-1} / 10^{-3} \mathrm{~m}^{3} \mathrm{~mol}^{-1}$. Bottom scale: in-vacuo wavenumber $\tilde{v} / \mu \mathrm{m}^{-1}$. Spectra at three temperatures $(T=298 \mathrm{~K}, 188 \mathrm{~K}$, and $78 \mathrm{~K}$; increasing sharpness) are shown (. . .). The individual Gaussians $\left(\mathrm{G}_{i}\right)$ and their sum are shown (-). Vertical lines (. . .) indicate the fitted data range. (a) Gaussian band analysis of the spectra of fully oxidised grey Desulfovibrio desulfuricans DFx in sodium cacodylate buffer pH 7.0, $I=30 \mathrm{~mm}$ mixed with glycerol, $1: 2$ (v/v). (b) Gaussian band analysis of the spectra of fully oxidised grey Desulfovibrio desulfuricans DFx in calcium cacodylate buffer $\mathrm{pH} 7.0, I=30 \mathrm{~mm}$ mixed with glycerol, $1: 2(\mathrm{v} / \mathrm{v})$.

$\pm 1 / 3$, the sign of $D$ cannot be determined by an EPR experiment. Sometimes in the literature the case $E / D=1 / 3$ has been called "isotropic", but the zero field splitting is always isotropic and the external magnetic field splitting is only isotropic when $D$ and $E$ vanish, i.e. in cubic or icosahedral symmetry.

EPR-spectra of either DFx centre in oxidised form were simulated using a published PC-programme ${ }^{31}$ and optimised parameters from previous work. ${ }^{6,11,17}$ The generic parameters of oxidised $\mathrm{Fe}_{\mathrm{I}}$ were $D=0.75 \mathrm{~cm}^{-1}$ and $E / D=0.083$. The simulated EPR-spectrum of oxidised $\mathrm{Fe}_{\mathrm{II}}$ was similarly generated using $D=-1.40 \mathrm{~cm}^{-1}$ and $E / D=-0.31$. This value of the $D$ parameter was found from Mössbauer spectral data. ${ }^{11}$ In the absence of an external magnetic field the three doublets are equally spaced by the amount $4 \sqrt{7} \mathrm{D} / 3=4.94 \mathrm{~cm}^{-1}$. Diagonalisation of the energy matrix of the spin Hamiltonian, eqn. (1), within the ${ }^{6} \mathrm{~A}$ manifold shows that the resonance at $g_{\text {eff }} \approx 4.3$ is due to a transition within the middle doublet, and that it is virtually isotropic up to $\boldsymbol{B} \approx 1 \mathrm{~T}$ but not beyond.

\section{Temperature dependent optical charge transfer bandshapes}

Clues to the electronic-vibrational coupling pattern of the CT bands of DFx and possible structural inhomogeneity come
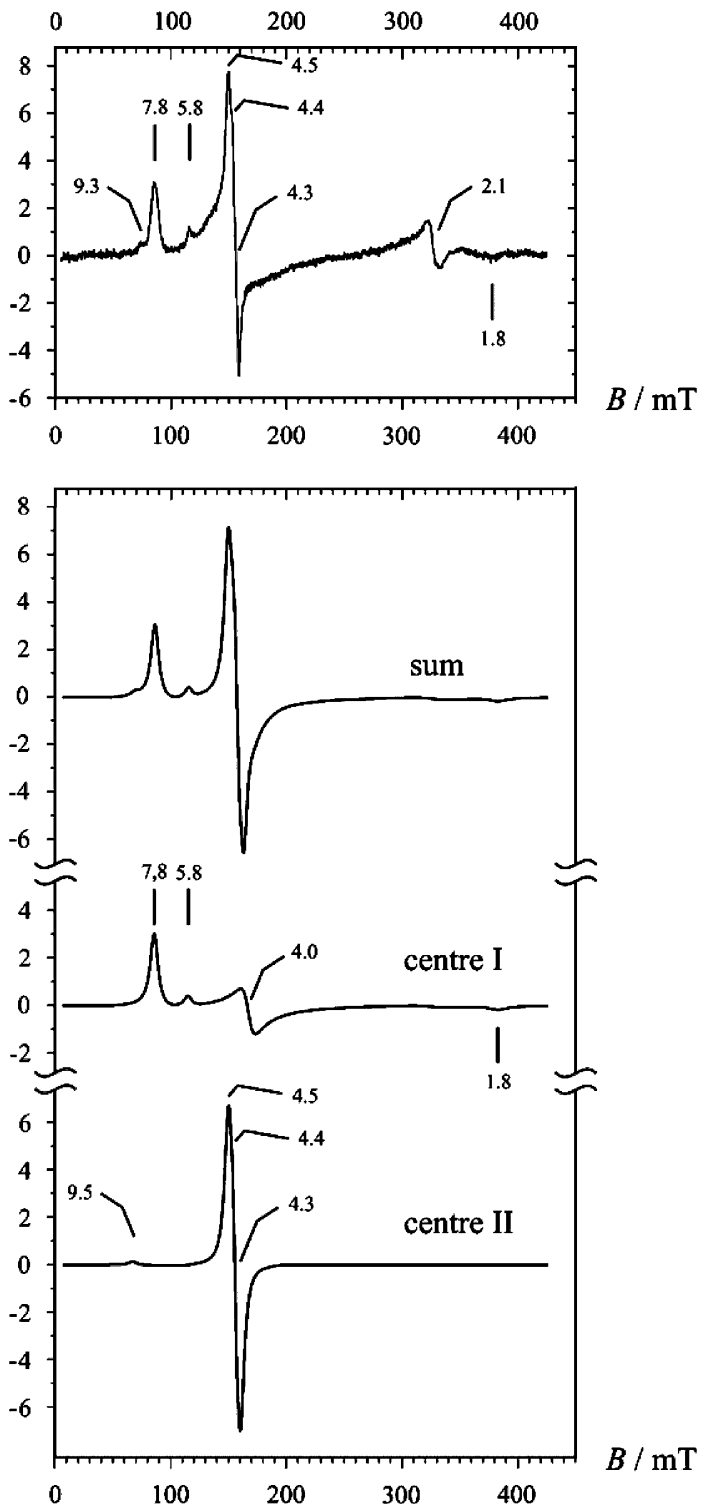

Fig. 7 Upper panel: the EPR spectrum of fully oxidized grey Desulfovibrio desulfuricans DFx in sodium cacodylate buffer $\mathrm{pH} 7.0, I=$ $30 \mathrm{~mm}$ mixed with glycerol, $1: 2(\mathrm{v} / \mathrm{v}) . T=8.2 \mathrm{~K}$, microwave frequency $v=9.403 \mathrm{GHz} . g_{\text {eff }}=9.11,7.88,5.8, \approx 4$ (clearly of composite nature), 1.8. The signal at $g_{\text {eff }}=2.1$ is due to a slight excess of $\left[\mathrm{IrCl}_{6}\right]^{2-}$ used to ensure that DFx was completely oxidised. Lower panel: simulated EPR spectra: ${ }^{31}$ Top: sum of EPR spectra, each simulated by the spinHamiltonian, eqn. (1), within the ${ }^{6} \mathrm{~A}$ ground term of $\mathrm{Fe}(\mathrm{III})$. Middle: simulation of EPR spectrum of centre I, $\mathrm{Fe}_{\mathrm{I}}(\mathrm{III}): g_{\text {eff }}=7.8,5.8, \approx 4.0$, and 1.8. Spin-Hamiltonian parameters $E / D=0.062 / 0.75=0.083$. Bottom: simulation of EPR of centre II, $\mathrm{Fe}_{\mathrm{II}}$ (III): $g_{\text {eff }}=9.3, \approx 4.3$ (with fine structure); Spin-Hamiltonian parameters $\varepsilon / D=0.43 /(-1.40)=-0.31$.

from multiphonon absorption bandshape analysis. We shall use the formalism below, which is appropriate for strong electronicvibrational couplings and the basis for the bandshape analysis in the next section. ${ }^{2,21,32}$

The molar absorption coefficient $\varepsilon$ at the frequency $v$, is given by

$$
\varepsilon(v)=\frac{4 \pi}{3 c} h v K_{\mathrm{eg}}(v)
$$

where $c$ is the speed of light in the solvent and $h$ Planck's constant. The bandshape functions for CT between the ground

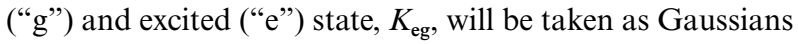

$$
K_{\mathrm{eg}}(v)=2 \frac{\sqrt{\pi}}{\Delta_{\mathrm{env}}}\left(M_{\mathrm{eg}}\right)^{2} \exp \left(-\frac{\left(h v-h v_{\max }\right)^{2}}{\Delta_{\mathrm{env}}^{2}} 4 \ln 2\right)
$$


where $v_{\max }$ is the frequency at the Gaussian maximum, $\Delta_{\text {env }}$ the full width at half maximum (FWHM) for homogeneous environmental broadening, and $M_{\mathrm{eg}}$ the electronic transition dipole. The whole CT spectrum is represented by sums of individual $K_{\mathrm{eg}}(v)$ (eqn. (4)) inserted into eqn. (3).

Eqn. (3) and (4) imply the following about the mechanism of the transition:

a) The analysis includes the absorption frequency in the pre-exponential factor (eqn. (3)). This affects the bandshape parameters for broad absorption bands such as for grey and pink DFx.

b) Electronic-vibrational coupling to a manifold of continuous, linear solvent or protein modes, or to local harmonic low frequency modes is the dominating broadening mechanism. Specific nuclear modes are metal-ligand stretching modes, but many of the bandwidths correspond to much larger reorganization free energies than for coupling solely to these modes in thermal ET. ${ }^{33}$ Significantly larger bond reorganization, as well as protein and solvent reorganization are therefore likely to accompany the optical transitions. Conversion between different conformational states, such as suggested from Mössbauer spectroscopy ${ }^{11}$ could also be important for some of the transitions.

c) Bandshape features determined by excited state lifetimes are disregarded. This would give Lorentzian or Voigtian spectral profiles.

d) A suitable, homogeneous bandwidth form is ${ }^{2,21}$

$$
\Delta_{\mathrm{env}}^{2}=\frac{2 E_{\mathrm{r}}}{\ln 2} \hbar \omega_{\mathrm{m}} \operatorname{coth}\left(\frac{\hbar \omega_{\mathrm{m}}}{2 k T}\right)
$$

where $\omega_{\mathrm{m}}$ is the frequency at the maximum of the nuclear frequency distribution coupled to the transition, $E_{\mathrm{r}}$ the overall nuclear reorganization free energy (electronic-vibrational coupling factor), $k$ Boltzmann's constant, and $T$ the temperature. The relation between the energy maximum of the optical transition, $h v_{\max }$, and $E_{\mathrm{r}}$, is (Fig. 8)

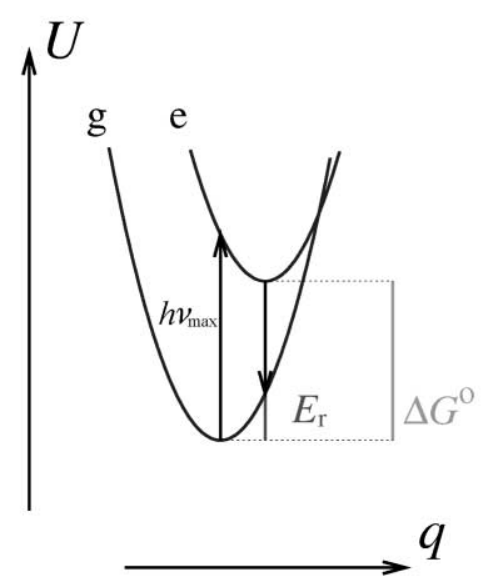

Fig. 8 Potential Gibbs free energy surfaces of the ground "g" and excited state "e" of optical CT transitions. The free energy gap, $\Delta G^{\circ}$, and total nuclear reorganisation energy $E_{\mathrm{r}}$ (local and environmental) corresponding to eqn. (4) are shown.

$$
h v_{\max }=E_{\mathrm{r}}+\Delta G^{\circ}
$$

where $\Delta G^{\circ}$ is the thermodynamic free energy gap between the ground and excited states.

e) The bandwidth takes the following limiting forms at high ("h") and low ("l") temperatures

$$
\Delta_{\mathrm{env}, \mathrm{h}}^{2}=\frac{4}{\ln 2} E_{\mathrm{r}} k T
$$

$$
\Delta_{\mathrm{env}, \mathrm{l}}^{2}=\frac{2}{\ln 2} E_{\mathrm{r}} \hbar \omega_{\mathrm{m}}
$$

$\Delta_{\text {env }}$ thus depends linearly on $\sqrt{ } T$ at high $T$ and is independent of $T$ at low $T$. Eqn. (7a) and (7b) represent different physical behaviour of the nuclear displacement, i.e. thermal activation in the high- $T$ limit, and activationless nuclear tunnelling in the low- $T$ limit.

f) Inhomogeneous broadening and distributions of conformational states are also formally represented by eqn. (4) and (5). The width then represents the conformational distribution, convoluted with homogeneous Gaussian broadening. Conformational mode freezing around the glass transition temperature rather than transition between nuclear tunnelling and thermal activation is then a feature of the $T$-pattern of the bandwidth. This seems to be the case for several transitions in grey and pink DFx.

g) Eqn. (3)-(7) constitute a formal frame adequate for grey and pink DFx, rather independently of the broadening mechanism. The frame can be extended to multi-mode dynamics, anharmonic modes, etc. as warranted. ${ }^{2,21,32}$ Harmonic local modes would be particularly the metal-ligand $\left(250-400 \mathrm{~cm}^{-1}\right)$ and Cys-S $-\mathrm{C}_{\beta}$ stretching modes, $c f$. below. These would be incorporated in the homogeneous bandwidths at room temperature. At low temperatures they would be converted to vibrational progressions broadened by the protein/solvent. Since the protein/solvent broadening is strong and the many electronic transitions already need a large number of parameters the vibrational progressions can be regarded as part of the temperature dependent homogeneous bandwidth in eqn. (5) and (7) which also represent the nuclear tunnelling behaviour inherent in the vibrational progression.

\section{Charge transfer bandshape analysis and vibronic coupling patterns of grey $\mathrm{DFx}$}

The following analysis is based on a 27-parameter system comprising eight Gaussians (no. 1-8) accounting for the CT region of grey $\mathrm{DFx}$ in $\mathrm{NaCcd}$ and $\mathrm{CaCcd}$ buffers, respectively, in addition to an extra Gaussian (no. 0) accounting for the near-UV $\left(\pi^{*} \longleftarrow \pi\right)$ band wing (Fig. 6a and b). This numerical system converged when applied to each of the 45 recorded spectra, beginning with the spectrum for the lowest temperature, resulting in $27 \times 45$ parameters. With this number of parameters numerical instability was occasionally encountered for the spectra at higher temperatures. The wavelengths of all band maxima depend weakly on the temperature. This excludes the fact that vibrational frequency changes and other nonlinear electronic-vibrational features are important. Focus is instead on the temperature dependence of the bandwidths.

DFx charge transfer spectra in sodium cacodylate buffer. The bandshape parameters are collected in Table 2, and Fig. 9a shows the $T$-dependence of the CT bandwidths. The "zeroth" Gaussian included to account for the absorption of aromatic residue band wing ensured convergence of the 27 spectralparameter system for each of the 45 spectra. The data prompt the following observations:

a) The RT bandwidths span a wide range from 1500 to 5000 $\mathrm{cm}^{-1}$. The $T$-dependence seems to fall into three kinds of behaviour. The widths of $\mathrm{G}_{5}$ and $\mathrm{G}_{8}$ exhibit a pronounced increase as the temperature is raised. $\mathrm{G}_{8}$ is a component of the $641 \mathrm{~nm} \mathrm{Fe}$ II assigned RT band. The very broad band at $641 \mathrm{~nm}$ seems to hold two Gaussian components, $G_{7}$ and $G_{8}$, with rather different $T$-patterns. The $\mathrm{G}_{5}$ and $\mathrm{G}_{8} \mathrm{RT}$ widths are 4493 and $3813 \mathrm{~cm}^{-1}$, respectively. $E_{\mathrm{r}}$ can be estimated to 8800 and $6530 \mathrm{~cm}^{-1}$, respectively. The corresponding coupling frequencies are 150 and $170 \mathrm{~cm}^{-1}$. The low coupling frequencies and strong temperature dependence indicate strong coupling to 

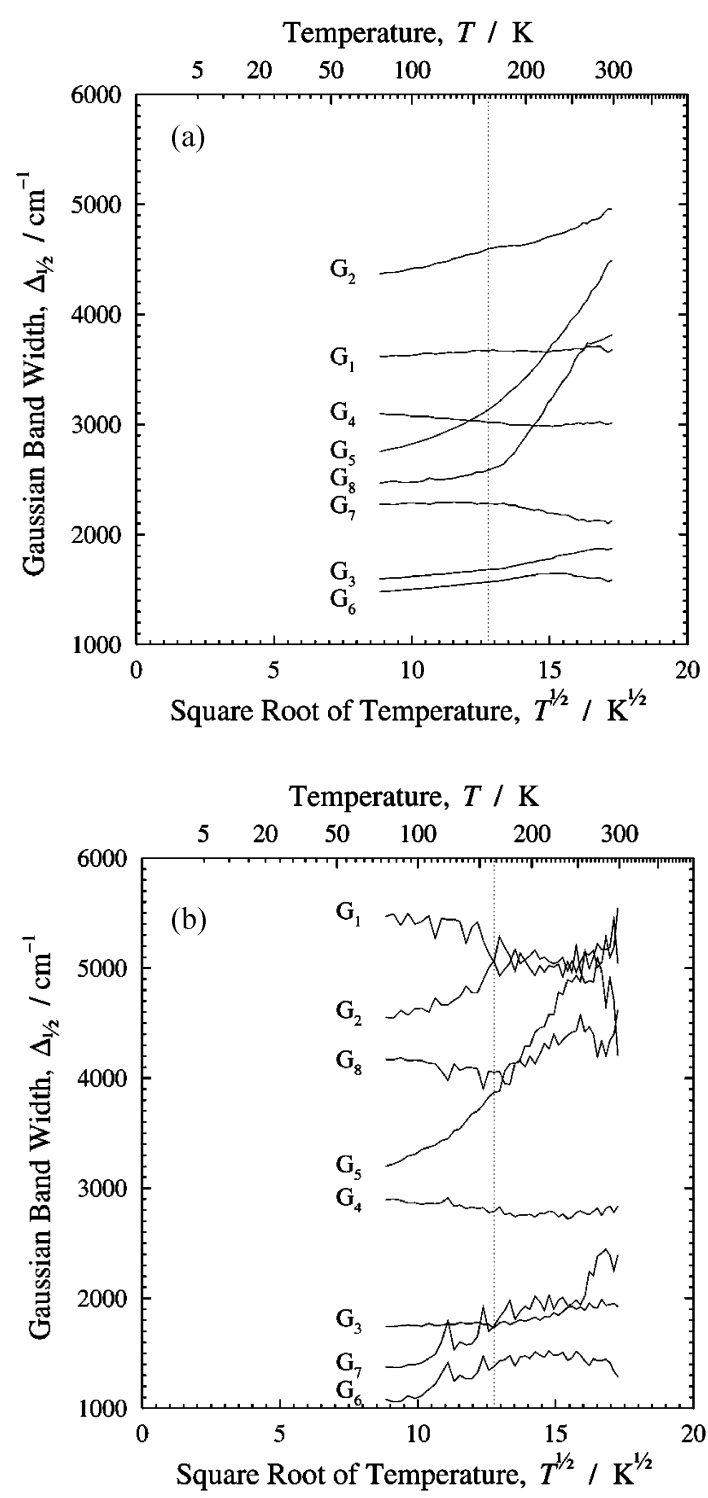

Fig. 9 (a) Temperature dependence of the bandwidths in the Gaussian analysis (Fig. 6a) for the sodium cacodylate buffer case. Top scale: temperature $T / \mathrm{K}$. Left scale: Gaussian bandwidth at half maximum (FWHM) $\Delta_{1 / 2} / \mathrm{cm}^{-1}$. Bottom scale: square root of temperature $\sqrt{T} / \sqrt{\mathrm{K}}$ The vertical line indicates the glass transition temperature $T_{\mathrm{g}}=163 \mathrm{~K}$ of the glycerol/buffer mixture. Each band is denoted with a $\mathrm{G}_{i}$ corresponding to the Gaussians in Fig. 6a and Table 2. (b) Temperature dependence of the bandwidths in the Gaussian analysis (Fig. 6b) for the calcium cacodylate buffer case. Top scale: temperature $T / \mathrm{K}$. Left scale: Gaussian bandwidth at half maximum (FWHM) $\Delta_{1 / 2} / \mathrm{cm}^{-1}$. Bottom scale: square root of temperature $\sqrt{ } T / \sqrt{ } \mathrm{K}$. The vertical line indicates the glass transition temperature $T_{\mathrm{g}}=163 \mathrm{~K}$ of the glycerol/buffer mixture Each band curve is denoted with a $\mathrm{G}_{i}$ corresponding to the Gaussians Fig. $6 \mathrm{~b}$ and Table 3.

solvent or protein modes, with lower frequencies than local molecular modes.

b) The second group comprises $G_{2}$ and $G_{3}$ assigned to $F_{1}$ which show a moderate but systematic bandwidth increase of $\approx 15 \%$ in the temperature range applied. The limiting $\mathrm{G}_{2}$ width at low $T$ approaches more than $4000 \mathrm{~cm}^{-1}$. This calls for inhomogeneous broadening mechanisms and inclusion of conformational sub-states of DFx whereas the $\mathrm{G}_{3} 1597 \mathrm{~cm}^{-1}$ width accords with homogeneous broadening. The weak $T$-dependence indicates that higher coupling frequencies are important.

c) The $T$-dependence of the widths of $\mathrm{G}_{1}\left(\mathrm{Fe}_{\mathrm{II}}\right), \mathrm{G}_{4}\left(\mathrm{Fe}_{\mathrm{I}}\right), \mathrm{G}_{6}$ $\left(\mathrm{Fe}_{\mathrm{I}}\right.$ and $\left.\mathrm{Fe}_{\mathrm{II}}\right)$, and $\mathrm{G}_{7}\left(\mathrm{Fe}_{\mathrm{II}}\right) \mathrm{nm}$ is clearly weaker. This indicates that both inhomogeneous broadening and coupling to highfrequency local modes could be important. The almost complete $T$-independence is, however, puzzling, as protein and other soft modes would be expected to contribute significantly in addition to local modes around the metal centres, $c f$. below. The weakly "inverse" $T$-dependence of the $\mathrm{G}_{6}$ and $\mathrm{G}_{7}$ bandwidths is straightforwardly in keeping with the formalism but the effect is too small for specific conclusions. The critical vibrational frequency, which would control the transition between nuclear mode displacement by thermal activation and by nuclear tunnelling, would be in the range 280 to $430 \mathrm{~cm}^{-1}$ which is the range of the iron-Cys stretching frequencies. ${ }^{11}$ From local mode reorganisation energies for thermal ET from DFT calculations ${ }^{33}$ these modes only contribute $\approx 0.15 \mathrm{eV}$ or 1200 to $1300 \mathrm{~cm}^{-1}$ but this could be larger for the optical transition where cysteinyl radical formation is involved. Contributions from the higher-frequency $700 \mathrm{~cm}^{-1}$ Cys-S- $\mathrm{C}_{\beta}$ stretching mode can also be envisaged as this mode shows a strong resonance Raman signal on excitation in the CT region. ${ }^{11}$

The apparent electronic-vibrational couplings, i.e. the optical nuclear reorganization free energies, are about $1.09 \mathrm{eV}(8800$ $\left.\mathrm{cm}^{-1}\right)$ for $\mathrm{G}_{5}\left(\mathrm{Fe}_{\mathrm{I}}\right)$ and $1.33 \mathrm{eV}\left(10700 \mathrm{~cm}^{-1}\right)$ for $\mathrm{G}_{2}\left(\mathrm{Fe}_{\mathrm{I}}\right.$ and $\left.\mathrm{Fe}_{\text {III }}\right)$. It is $\approx 0.73 \mathrm{eV}$ for the $\mathrm{G}_{1}\left(\mathrm{Fe}_{\mathrm{II}}\right)$ and $0.81 \mathrm{eV}$ for $\mathrm{G}_{8}\left(\mathrm{Fe}_{\mathrm{II}}\right)$. They are smaller, 0.14 to $0.49 \mathrm{eV}$ for $\mathrm{G}_{3}\left(\mathrm{Fe}_{\mathrm{I}}\right), \mathrm{G}_{4}\left(\mathrm{Fe}_{\mathrm{I}}\right), \mathrm{G}_{6}$ $\left(\mathrm{Fe}_{\mathrm{I}}\right.$ and $\left.\mathrm{Fe}_{\mathrm{II}}\right)$, and $\mathrm{G}_{7}\left(\mathrm{Fe}_{\mathrm{II}}\right)$. Most of these values are close to values for homogeneous broadening of single transitions accompanied by local mode and protein reorganization. The widths of $\mathrm{G}_{5}\left(\mathrm{Fe}_{\mathrm{I}}\right)$ and $\mathrm{G}_{2}\left(\mathrm{Fe}_{\mathrm{I}}\right.$ and $\left.\mathrm{Fe}_{\mathrm{II}}\right)$, which approach or exceed $1 \mathrm{eV}$ are, however, unphysical for homogeneously broadened transitions and must reflect temperature dependent conformational equilibria, distributions of conformational states, or more than a single transition. The notion of conformational equilibria for DFx was introduced early based on Mössbauer and other spectroscopy. ${ }^{11}$

The following other observations are appropriate:

d) The transitions between low- and high-temperature behaviour in Fig. 9a appear around the glass transition temperature of the glycerol-water solvent, $T_{\mathrm{g}} \approx 163 \mathrm{~K}$. The CT transitions of grey DFx therefore suggest that protein/solvent melting around the glass transition is reflected strongly in the transitions particularly corresponding to the $\mathrm{G}_{5}\left(\mathrm{Fe}_{\mathrm{I}}\right)$ and $\mathrm{G}_{8}$ $\left(\mathrm{Fe}_{\mathrm{II}}\right)$ Gaussians.

e) The CT widths of the $\mathrm{G}_{5}\left(\mathrm{Fe}_{\mathrm{I}}\right)$ and $\mathrm{G}_{8}\left(\mathrm{Fe}_{\mathrm{II}}\right)$ Gaussians follow roughly a $\Delta_{\text {env }} / \sqrt{ } T$-dependence above the transition temperature. This accords with conformational relaxation, but formally also with homogeneous broadening in local lowfrequency modes. As noted, the bandwidths are, however, far too large for the latter mechanism.

In conclusion all the CT bands of grey DFx in NaCcd buffer display strong electronic-vibrational coupling. The widths and the temperature dependence for the $\mathrm{G}_{3}\left(\mathrm{Fe}_{\mathrm{I}}\right), \mathrm{G}_{6}\left(\mathrm{Fe}_{\mathrm{I}}\right.$ and $\left.\mathrm{Fe}_{\mathrm{II}}\right)$, and $\mathrm{G}_{7}\left(\mathrm{Fe}_{\mathrm{II}}\right)$ Gaussians are small enough to accord with homogeneous broadening and significant homogeneous collective protein and solvent mode coupling (the $408 \mathrm{~nm} \mathrm{CD}\left(\mathrm{Fe}_{\mathrm{I}}\right)$ band, the $565 \mathrm{~nm}\left(\mathrm{Fe}_{\mathrm{I}}\right)$ band and the $610 \mathrm{~nm}\left(\mathrm{Fe}_{\mathrm{II}}\right) \mathrm{CD}$ feature). The widths of the other Gaussians are so large that broad conformational distributions and/or monomer-dimer equilibria must be involved. Both these features depend on the temperature and are coupled to the solvent structural changes such as noted by the bandwidth features at the glass transition temperature.

The CT bands of pink DFx display similar temperature dependent broadening features but these bands are not well resolved compared to those of grey DFx even at the lowest temperature $(78 \mathrm{~K})$. We do not, therefore, provide a bandshape analysis for pink DFx.

Analysis of DFx charge transfer spectra in calcium cacodylate buffer. $\mathrm{Ca}^{2+}$-ions are indispensable for crystallisation of DFx. ${ }^{9}$ With a view to illuminating the role of $\mathrm{Ca}^{2+}$ in the conformational dynamics of the protein and the monomer-dimer DFx equilibrium the same spectral recording and band- 
shape analysis as above was undertaken for grey DFx in CaCcd buffer.

The spectra and the Gaussian bandwidths (Figs. 6b, 9b and Table 3) exhibit basically the same features and transitions as for grey DFx in NaCcd buffer, Figs. 3 and 9a. Eight Gaussians together with one Gaussian for the protein-band, fit the spectra in the whole CT region for all 45 applied temperatures. Positions of the maxima and intensities and widths of the Gaussian bands are similarly summarised in Table 3. The following differences of the grey DFx spectrum in CaCcd buffer, where the protein is to a certain extent in the dimer form, compared with $\mathrm{NaCcd}$ buffer can be distinguished:

a) $\mathrm{G}_{1}$ at $318 \mathrm{~nm}\left(\mathrm{Fe}_{\mathrm{I}}\right.$ and $\left.\mathrm{Fe}_{\mathrm{II}}\right)$ and $\mathrm{G}_{8}$ at $677 \mathrm{~nm}\left(\mathrm{Fe}_{\mathrm{II}}\right)$ in $\mathrm{NaCcd}$ buffer are blue-shifted to 313 and $635 \mathrm{~nm}$, respectively, while $\mathrm{G}_{2}$ at $366 \mathrm{~nm}\left(\mathrm{Fe}_{\mathrm{I}}\right)$ is red-shifted to $370 \mathrm{~nm}\left(\mathrm{Fe}_{\mathrm{I}}\right)$ in $\mathrm{CaCcd}$ buffer. The $677 \mathrm{~nm}\left(\mathrm{Fe}_{\mathrm{II}}\right)$ band is not resolved such as for $\mathrm{NaCcd}$ buffer but the large width is strongly indicative that more than one transition is involved.

b) The temperature dependence of the bandwidths accord largely with the observations for $\mathrm{NaCcd}$ buffer. The $\mathrm{G}_{1}\left(\mathrm{Fe}_{\mathrm{I}}\right.$ and $\left.\mathrm{Fe}_{\mathrm{II}}\right), \mathrm{G}_{4}\left(\mathrm{Fe}_{\mathrm{I}}\right)$, and $\mathrm{G}_{8}\left(\mathrm{Fe}_{\mathrm{II}}\right)$ Gaussians are, however, all notably broader than in $\mathrm{NaCcd}$ buffer.

c) The effects of $\mathrm{Ca}^{2+}$-ions on grey $\mathrm{DFx}$ in aqueous buffer solution seem to induce some conformational freezing but broad inhomogeneous distributions of "frozen" conformations remain. This may be related to a shift in the monomer-dimer equilibrium as reflected in the strong relaxational features of two of the Gaussians at the glass transition temperature, i.e. $\mathrm{G}_{5}\left(\mathrm{Fe}_{\mathrm{I}}\right)$ and $\mathrm{G}_{7}\left(\mathrm{Fe}_{\mathrm{I}}\right.$ and $\left.\mathrm{Fe}_{\mathrm{II}}\right)$.

\section{Conclusion}

We have provided a comprehensive spectroscopic investigation of the two-centre modular non-heme redox protein DFx from Desulfovibrio desulfuricans. The investigation rests on variabletemperature UV/VIS absorption spectroscopy and bandshape analysis, $\mathrm{MCD}$, and CD spectroscopy, and EPR spectroscopy of DFx in all the three oxidation states (EPR only for grey DFx). The spectroscopic characterisation has enabled disclosure particularly of the electronic-vibrational coupling features, and conformational relaxation patterns, which accompany all the $\mathrm{CT}$ transitions in the visible, near-UV, and NIR spectral regions of grey and pink DFx. Other issues addressed are the electronic coupling between $\mathrm{Fe}_{\mathrm{I}}$ and $\mathrm{Fe}_{\mathrm{II}}$, and the deconvolution of local molecular and homogeneous protein/solvent nuclear motion from conformational inhomogeneous broadening caused by the protein and solvent, and conformational and monomer-dimer equilibria between different molecular species.

Absorption spectral recordings at variable temperatures have disclosed several grey and pink DFx CT bands in the visible spectral region, which are congested by thermal broadening at higher temperatures. This has enabled a detailed bandshape resolution in the whole temperature range from 78 to $298 \mathrm{~K}$. The minimal band assignment pattern discloses eight $\mathrm{CT}$ bands involving the metal centres. To this comes the strong $280 \mathrm{~nm}$ ( $\pi^{*} \longleftarrow \pi$ ) band that is vibrationally resolved at the lowest temperatures. The following observations refer to the CT bands. The best resolution was obtained for grey DFx, and most of the discussion refers to this form.

All the $\mathrm{CT}$ bands can be assigned to $\left(\mathrm{Fe}^{3+} \leftarrow \mathrm{S}^{-}\right)$transitions in oxidised $\mathrm{Fe}_{\mathrm{I}}$ and $\mathrm{Fe}_{\mathrm{II}}$. The central electronic process thus involves photo-induced formation of a $\mathrm{Cys}-\mathrm{S}^{*}-\mathrm{Fe}$ (II) unit in the excited electronic state(s). This implies that the accompanying nuclear reorganisation Gibbs free energy can be significantly larger than for thermal electron transfer processes of $\mathrm{Fe}_{\mathrm{I}}$ and $\mathrm{Fe}_{\mathrm{II}}$. The $\mathrm{Fe}-\mathrm{S}$ bonds are thus left intact in the latter case apart from minor bond adjustments. The optically induced reorganisation Gibbs free energies are expected to exceed significantly the values of 0.15 to $0.2 \mathrm{eV}$ estimated for thermal electron transfer on the basis of quantum chemical computations. ${ }^{33}$
The following conclusions referring to NaCcd buffer then emerge:

(i) Three of the Gaussians, i.e. $\mathrm{G}_{3}\left(\mathrm{Fe}_{\mathrm{I}}\right), \mathrm{G}_{6}\left(\mathrm{Fe}_{\mathrm{I}}\right)$ and $\mathrm{G}_{7}\left(\mathrm{Fe}_{\mathrm{II}}\right)$ accord with homogeneous electronic-vibrational couplings dominated by local nuclear modes $\left(E_{\mathrm{r}} \approx 0.2 \mathrm{eV}\right)$. The $\mathrm{G}_{6}$ and $\mathrm{G}_{7}$ widths are largely independent of the temperature, while the $G_{3}$ width increases moderately with increasing temperature. This suggests that there is strong electronic coupling to a highfrequency mode such as the $700 \mathrm{~cm}^{-1} \mathrm{Cys}-\mathrm{S}-\mathrm{C}_{\beta}$ stretching mode for the bands related to $G_{6}$ and $G_{7}$ and stronger coupling to lower-frequency modes such as metal-ligand stretching modes for the bands related to $\mathrm{G}_{3}\left(\mathrm{Fe}_{\mathrm{I}}\right)$.

(ii) Three other Gaussians, i.e. $\mathrm{G}_{1}\left(\mathrm{Fe}_{\mathrm{II}}\right), \mathrm{G}_{4}\left(\mathrm{Fe}_{\mathrm{I}}\right)$ and $\mathrm{G}_{8}$ $\left(\mathrm{Fe}_{\mathrm{II}}\right)$ exhibit RT widths of $0.3-0.4 \mu \mathrm{m}^{-1}$ or apparent nuclear reorganisation Gibbs free energies of about $1 \mathrm{eV}$. The temperature dependence of all the bands is weak compared to expectations from the theoretical bandshape formalism used. If about $0.5 \mathrm{eV}$ of nuclear reorganisation can be assigned as a maximum for homogeneous local and protein/solvent mode broadening corresponding to $0.2-0.25 \AA \mathrm{Fe}-\mathrm{S}$-Cys band stretch on optical CT excitation, then up to $0.2 \mathrm{eV}$ is left for other broadening mechanisms. The notable increase of the $\mathrm{G}_{8}\left(\mathrm{Fe}_{\mathrm{II}}\right)$ width around the solvent glass transition temperature and the approximate $\sqrt{ } T$-dependence above this temperature suggest that $T$-dependent conformational equilibria and conformational interactions between the monomers in temperaturedependent monomer-dimer equilibria is involved. Strong coupling to solvent-controlled conformational modes and relaxation of these modes as the glycerol/water solvent relaxes above the glass transition temperature is thus implied. This transition, therefore, appears to emerge as a sensitive probe for long-range and bulk conformational dynamic effects, in the glycerol/water/protein environment.

(iii) Two transitions are related to $\mathrm{G}_{2}$ and $\mathrm{G}_{5}$ (both $\mathrm{Fe}_{\mathrm{I}}$ ) that are particularly broad. These Gaussians are resolved components of even broader low-temperature bands. $\mathrm{G}_{2}$ and $\mathrm{G}_{5}$ may, however, be further composite as the MCD spectra seem to hold both $\mathrm{C}$ - and A-terms in this spectral region.

(iv) The overarching spectral effects of dimer structurepromoting $\mathrm{Ca}^{2+}$ ions on the local electronic features of the metal centres are small. Apart from minor blue- and red-shifts of the individual charge transfer bands the most conspicuous difference is in the temperature dependence of the $\mathrm{G}_{1}\left(\mathrm{Fe}_{\mathrm{II}}\right)$ (blue-shifted by $5 \mathrm{~nm}$ compared to the NaCcd buffer) bandwidth, which increases strongly with increasing temperature above the solvent glass transition temperature. Both centres appear sensitive to protein conformational relaxation features, which are in turn strongly coupled to the external solvent dynamics.

(v) The importance of conformational relaxation is, finally, notably illuminated by the changes in VT-MCD patterns of the $\left(\mathrm{Fe}^{3+} \longleftarrow \mathrm{S}^{-}\right)$-based transitions in grey and pink DFx. This is particularly apparent in the weaker and less well resolved bands in pink $\mathrm{DFx}$ in the $400-500 \mathrm{~nm}$ range associated with $\mathrm{Fe}_{\mathrm{I}}$.

\section{Acknowledgements}

Financial support from the Danish Natural Science and Technical Science Research Councils is acknowledged. We wish to give our special thanks to Frode Galsbøl who provided both $2\left\{\Lambda\right.$ - $\left.(+)_{\mathrm{D}}-\left[\mathrm{Co}(\mathrm{en})_{3}\right] \mathrm{Cl}_{3}\right\} \cdot \mathrm{NaCl} \cdot 6 \mathrm{H}_{2} \mathrm{O}$ and $\mathrm{K}_{2}\left[\mathrm{IrCl}_{6}\right]$ for our use.

\section{References and notes}

1 R. A. Marcus and N. Sutin, Biochim. Biophys. Acta, 1985, 811, 265-322.

2 J. Ulstrup and A. M. Kuznetsov, Electron Transfer in Chemistry and Biology, Wiley, Chichester 1999.

3 V. H. Teixeira, C. M. Soares and A. M. Baptista, J. Biol. Inorg. Chem., 2002, 7, 200-216. 
4 I. B. Coutinho and A. V. Xavier, Methods Enzymol., 1994, 243, $119-140$.

5 L. S. Conrad, J.-J. Karlsson and J. Ulstrup, Eur. J. Biochem., 1995, 231, 133-141.

6 I. Moura, P. Tavares, J. J. G. Moura, N. Ravi, B. H. Huynh, M.-Y. Liu and J. LeGall, J. Biol. Chem., 1990, 265, 21596-21602.

7 M. F. J. M. Verhagen, W. G. B. Voorhorst, J. A. Kolkman, R. B. G. Wolbert and W. R. Hagen, FEBS Lett., 1993, 336, 13-18.

8 D. Apiyo, K. Jones, J. Guidry and P. Wittung-Stafshede, Biochemistry, 2001, 40, 4940-4948.

9 A. V. Coelho, P. M. Matias, M. A. Carrondo, P. Tavares, J. J. G. Moura, I. Moura, V. Fülöp, J. Hadju and J. LeGall, Protein Science, 1996, 5, 1189-1191.

10 B. Devreese, P. Tavares, J. Lampreia, N. Van Damme, J. LeGall, J. J. G. Moura, J. Van Breumen and I. Moura, FEBS Lett., 1996, 385, 138-142.

11 P. Tavares, N. Ravi, J. J. G. Moura, J. LeGall, Y.-H. Huang, B. R. Crouse, M. K. Johnson, B. H. Huynh and I. Moura, J. Biol. Chem., 1994, 269, 10504-10510.

12 A. Coelho, P. Matias, V. Fülöp, A. Thompson, A. Gonzales and M. A. Carrondo, J. Biol. Inorg. Chem., 1997, 2, 680-689.

13 M. Archer, R. Huber, P. Tavares, I. Moura, J. J. G. Moura, M. A. Carrondo, L. C. Sieker, J. LeGall and M. J. Romão, J. Mol. Biol., 1995, 251, 690-702.

14 C. V. Romão, M. Y. Liu, J. LeGall, C. M. Gomes, V. Braga, I. Pacheco, A. Xavier and M. Teixeira, Eur. J. Biochem., 1999, 261, 438-443.

15 M. Lombard, M. Fontecave, D. Touati and V. Nivière, J. Biol. Chem., 2000, 275, 115-121.

16 E. D. Coulter, J. P. Emerson, D. M. Kurtz, Jr. and D. E. Cabelli, J. Am. Chem. Soc., 2000, 122, 11555-11556.

17 C. Ascenso, F. Rusnak, I. Cabrito, M. J. Lima, S. Naylor, I. Moura and J. J. G. Moura, J. Biol. Inorg. Chem., 2000, 5, 720-729.

18 A. P. Yeh, Y. Hu, F. E. Jenney, M. W. W. Adams and D. C. Rees, Biochemistry, 2000, 39, 2499-2508.
19 M. D. Clay, F. E. Jenney, Jr., P. L. Hagedoorn, G. N. George, M. W. W. Adams and M. K. Johnson, J. Am. Chem. Soc., 2002, 124, 788-805.

20 M. A. Carrondo, personal communication.

21 E. M. Itskovich, J. Ulstrup and M. A. Vorotyntsev, in The Chemical Physics of Solvation. Part B. Spectroscopy of Solvation., ed. R. R. Dogonadze, E. Kálmán, A. Kornyshev and J. Ulstrup, Elsevier, Amsterdam, 1986, ch. 6, pp. 223-310.

$22 \mathrm{http}: / /$ www.jasco-europe.com/trep.htm, Standards for $C D$, report 043. $2\left\{\Lambda-(+)_{\mathrm{D}}-\left[\mathrm{Co}(\mathrm{en})_{3}\right] \mathrm{Cl}_{3}\right\} \cdot \mathrm{NaCl} \cdot 6 \mathrm{H}_{2} \mathrm{O}$ was provided by, F. Galsbøl, Department of Chemistry, University of Copenhagen, synthesis unpublished.

23 I. Moura, A. V. Xavier, R. Cammack, M. Bruschi and J. LeGall, Biochim. Biophys. Acta, 1978, 533, 156-162.

24 V. S. Oganesyan, S. J. George, M. R. Cheesman and A. J. Thomson, J. Chem. Phys., 1999, 110, 762-777.

25 W. Lovenberg and B. E. Sobel, Proc. Natl. Acad. Sci. USA, 1965, 54 193-199.

26 I. A. Abreu, L. M. Saraiva, C. M. Soares, M. Teixeira and D. E. Cabelli, J. Biol. Chem., 2001, 276, 38995-39001.

27 T. Jovanović, C. Ascenso, K. R. O. Hazlett, R. Sikkink, C. Krebs, R. Litwiller, L. M. Benson, I. Moura, J. J. G. Moura, J. D. Radolf, B. H. Huynh, S. Naylor and F. Rusnak, J. Biol. Chem., 2000, 275, 28439-28448.

28 A. P. Yeh, Y. Hu, F. E. Jenney, Jr., M. W. W. Adams and D. C. Rees, Biochemistry, 2000, 39, 2499-2508.

29 A. J. Thomson, M. R. Cheesman and S. J. George, Methods Enzymol., 1993, 226, 199-232.

30 J. S. Griffith, Mol. Phys., 1964, 8, 213-216.

31 J. Glerup and H. Weihe, Acta Chem. Scand., 1991, 45, $444-448$.

32 A. M. Kjaer and J. Ulstrup, J. Am. Chem. Soc., 1987, 109, 1934-1942.

33 E. Sigfridsson, M. H. M. Olsson and U. Ryde, J. Phys. Chem. B, $2001, \mathbf{1 0 5}, 5546-5552$. 